\title{
Transcription analysis of neonicotinoid resistance in Mediterranean (MED) populations of $B$. tabaci reveal novel cytochrome P450s, but no nAChR mutations associated with the phenotype
}

Aris $\| l i a s^{1 \dagger}{ }^{1 \dagger}$ Jacques Lagnel ${ }^{2+}$, Despoina E. Kapantaidaki ${ }^{1,3}$, Emmanouil Roditakis $^{1}$, Costas S. Tsigenopoulos ${ }^{2}$, John Vontas ${ }^{4,5}$ and Anastasia Tsagkarakou ${ }^{1 *}$

\begin{abstract}
Background: Bemisia tabaci is one of the most damaging agricultural pests world-wide. Although its control is based on insecticides, B. tabaci has developed resistance against almost all classes of insecticides, including neonicotinoids.

Results: We employed an RNA-seq approach to generate genome wide expression data and identify genes associated with neonicotinoid resistance in Mediterranean (MED) B. tabaci (Q1 biotype). Twelve libraries from insecticide resistant and susceptible whitefly populations were sequenced on an Illumina Next-generation sequencing platform, and genomic sequence information of approximately $73 \mathrm{Gbp}$ was generated. A reference transcriptome was built by de novo assembly and functionally annotated. A total of 146 P450s, 18 GSTs and 23 CCEs enzymes (unigenes) potentially involved in the detoxification of xenobiotics were identified, along with 78 contigs encoding putative target proteins of six different insecticide classes. Ten unigenes encoding nicotinic Acetylcholine Receptors (nAChR), the target of neoinicotinoids, were identified and phylogenetically classified. No nAChR polymorphism potentially related with the resistant phenotypes, was observed among the studied strains.

DE analysis revealed that among the 550 differentially (logFC $>1$ ) over-transcribed unigenes, 52 detoxification enzymes were over expressed including unigenes with orthologues in P450s, GSTs, CCE and UDP-glucuronosyltransferases. Eight P450 unigenes belonging to clades CYP2, CYP3 and CYP4 were highly up-regulated (logFC > 2) including CYP6CM1, a gene already known to confer imidacloprid resistance in B. tabaci. Using quantitative qPCRs, a larger screening of field MED B. tabaci from Crete with known neonicotinoid phenotype was performed to associate expression levels of P450s with resistance levels. Expression levels of five P450s, including CYP6CM1, were found associated with neonicotinoid resistance. However, a significant correlation was found only in CYP303 and CYP6CX3, with imidacloprid and acetamiprid respectively.

(Continued on next page)
\end{abstract}

\footnotetext{
* Correspondence: tsagkarakou@nagref.gr

${ }^{\dagger}$ Equal contributors

"Hellenic Agricultural Organisation - "DEMETER", NAGREF - Institute of Olive

Tree, Subtropical Crops and Viticulture, Heraklion, Greece

Full list of author information is available at the end of the article
}

\section{Biomed Central}

(C) 2015 Ilias et al. Open Access This article is distributed under the terms of the Creative Commons Attribution 4.0 International License (http://creativecommons.org/licenses/by/4.0/), which permits unrestricted use, distribution, and reproduction in any medium, provided you give appropriate credit to the original author(s) and the source, provide a link to the Creative Commons license, and indicate if changes were made. The Creative Commons Public Domain Dedication waiver (http://creativecommons.org/publicdomain/zero/1.0/) applies to the data made available in this article, unless otherwise stated. 
(Continued from previous page)

Conclusion: Our work has generated new toxicological data and genomic resources which will significantly enrich the available dataset and substantially facilitate the molecular studies in MED B. tabaci. No evidence of target site neonicotinoid resistance has been found. Eight P450 unigenes, including CYP6CM1, were found significantly over-expressed in resistant B. tabaci. This study suggests at least two novel P450s (CYP303 and CYP6CX3) as candidates for their functional characterization as detoxification mechanisms of neonicotinoid resistance in B. tabaci.

Keywords: Bemisia tabaci, RNA-Seq, Neonicotinoids, Resistance mechanisms

\section{Background}

The whitefly Bemisia tabaci (Hemiptera: Aleyrodidae) (sweet potato or tobacco whitefly) is a broad phloem feeding herbivore. B. tabaci is a complex of 31 putative morphocryptic species [1] and one of the most damaging pests of protected, field crops and ornamentals worldwide [2]. The current global status of B. tabaci as a pest refers mainly to two species of the complex, MEAM1 (Middle East-Asia Minor I, formerly referred to as biotype B) and MED (Mediterranean, formerly referred to as biotype Q) which are both highly invasive and polyphagous (over 1000 host plants reported) and particularly detrimental by transmitting viruses which cause serious crop diseases.

Currently, the control of B. tabaci relies on insecticides. However, their extensive and chronic use has imposed strong selection pressures for resistance to many insecticide classes in B. tabaci, including neonicotinoids, which are the world market leaders of insecticides [3-7]. Neonicotinoids act by binding on insect nicotinic acetylcholine receptors (nAChR). Imidacloprid was the first neonicotinoid introduced in the market in 1991 followed by acetamiprid, thiamethoxam, thiacloprid and clothianidin [8].

Insecticide resistance is typically characterized by a variety of molecular aberrations, such as transcriptional changes, gene amplification and point mutations in coding regions, which in turn result in phenotypic novelties like increased rates of insecticide detoxification or reduced sensitivity of the target protein(s). The most significant mechanisms of resistance to neonicotinoid insecticides are decreased target-site sensitivity and increased metabolic detoxification by cytochrome P450 monooxygenages $[9,10]$. In $B$. tabaci, it was shown that the cytochome P450 CYP6CM1 is the major resistance mechanism, as the gene expression is highly associated with the resistance phenotype in a number of populations worldwide [11], and it encodes a protein capable of metabolising neonicotinoids (such as imidacloprid and thiacloprid) to less toxic metabolites [10].

The phenomenon of insecticide resistance is particularly striking in Mediterranean countries, where suitable climatic conditions allow year-round crop production and the survival of $B$. tabaci $[3,12]$. Highly resistant MED populations have been reported in Crete, a major vegetable crop production area in Greece, especially towards the neonicotinoid insecticide imidacloprid [4]. Pymetrozine, pyriproxifen and spiromesifen, have been introduced and used in rotation with neonicotinoids, alongside an extensive use of natural enemies in whitefly control programs in the last 5 years in Crete. However, a recent resistance monitoring study from various locations in Southern Crete in 2012, showed that neonicotinoid resistance is still present in Crete [13].

The B. tabaci cytochome P450 CYP6CM1 was found to be over-expressed in Cretan populations; however, in some cases resistance ratios were not strongly correlated with CYP6CM1 expression level, suggesting that additional neonicotinoid resistance mechanisms may operate in Crete [13].

RNA sequencing (RNA-Seq) was the decisive tool to perform de novo transcriptome sequencing and has already been applied in transcriptome analysis of insects, such as Aedes aegypti, Anopheles funestus, Nilaparvata lugens, Locusta migratoria, B. tabaci, Trialeurodes vaporariorum [14-19] and mites [20], providing not only a better understanding of their biological functions at the molecular level but also genomic recourses on detoxification and target genes putatively involved in insecticide resistance $[19,21]$. Some recent studies have utilized these techniques to explore differences between resistance phenotypes in insects of medical importance such as $A$. aegypti [22] and Cimex lectularius [23]. In B. tabaci, there is a number of transcriptome resources generated by Roche 454 pyrosequencing or Illumina deep sequencing which have substantially improved our understanding on topics such as; symbiosis, virus-vector interactions, Bauveria bassiana infection, gut role or gene expression in different developmental stages and genetic groups within $B$. tabaci [15, 24-28]. To date only one study dealt with differential gene expression in strains with different resistant phenotypes [29]. Moreover, only a small percentage of the $B$. tabaci transcriptome data available explores the transcriptome of the MED lineage of this complex species [15, 28].

In the present study bioassays and transcriptome data from the MED species were analyzed to further characterize the underlying molecular mechanism responsible for neonicotinoid resistance in $B$. tabaci 
from Crete. Twelve libraries from three resistant and one susceptible B. tabaci strain including biological replicates were sequenced with the Illumina platform, and generated about $73 \mathrm{Gbp}$ of sequence data. This high throughput sequencing technique has allowed for global expression profiling of neonicotinoid resistant and susceptible populations. A reference transcriptome was built by de novo assembly and functionally annotated. It was then used to map the reads from insecticide-susceptible and resistant strains, study the levels of gene expression and gain genetic information for resistance mechanisms.

\section{Results and Discussion}

\section{Resistant phenotypes towards neonicotinoids}

Based on recent resistance monitoring in Cretan $B$. tabaci MED, we chose the whitefly populations used in the present study from those displaying the highest resistance phenotypes and heaviest neonicotinoid treatment history. Three field derived strains originally collected from eggplants maintained in the laboratory on cotton plants under Imidacloprid (GR0-IS, GR9-IS) or Acetamiprid Selection (GR4-AS) for few (six) generations were used in this study, along with their Parental 'relaxed' strains (GR0-IP, GR9-IP and GR4-AP), namely the respective original strains maintained in the absence of any insecticide contact. All comparisons were performed with a local susceptible reference strain (S-GR6), collected in Crete in 2006, in order to access differences at the transcription level linked with differences at the resistance phenotypes.

Mortality data and Resistance Ratios (RR) to the three neonicotinoid insecticides imidacloprid, acetamiprid and thiachloprid were determined by classical leaf dip bioassays on adult females (Table 1). Compared to the reference susceptible strain S-GR6, RR ranged from 9 to 56-fold for imidacloprid, from 4 to 20-fold for acetamiprid and >1000-fold for thiacloprid. Resistance ratios in the presence of piperonyl butoxide (PBO), an inhibitor of monooxygenases, were also investigated. Imidacloprid toxicity was increased in all strains (lethal concentration - $\mathrm{LC}_{50}$ was reduced from 423 to $185 \mathrm{mg} / \mathrm{L}$ for GR4-AS, from 301 to $207 \mathrm{mg} / \mathrm{L}$ for GR9-IS and from 177 to $52 \mathrm{mg} / \mathrm{L}$ for GR0-IS) after pre-exposure to PBO (Table 1), indicating that cytochrome $\mathrm{P} 450(\mathrm{~s})$ are involved in the reduced susceptibility against imidacloprid. The absence of complete suppression of resistance after pre-exposure to $\mathrm{PBO}$ treatment could indicate either the involvement of another resistance mechanism or the limited capacity of the use of PBO to detect $\mathrm{P} 450$ based insecticide resistance [30].

Compared to their relaxed parental strains, the respective selected strains exhibited higher resistance levels towards acetamiprid and imidacloprid but not towards thiacloprid, indicating that the mechanism underlying resitance to thiacloprid is not selected by imidacloprid or acetamiprid treatments.

The higher resistance levels against neonicotinoids were exhibited by strains GR4-AS and GR9-IS, each selected with a different neonicotinoid (acetamiprid and imidacloprid respectively). These two strains were therefore chosen for styding their transcriptomic profile in the RNA-seq experiment in comparison to the susceptible S-GR6, which was used as a reference strain. In order to better understand the part of B. tabaci transcriptom changes linked to the resistance, we compared also strains with the same genetic background which differ only in their resistant level. The pair GR4-AP and GR4-AS displayed the higher differences (6x with imidacloprid and $3.6 \times$ with acetamiprid) among the three selected-parental pairs of strains (Table 1). Therefore the parental strain GR4-AP was also included in the RNA-seq experiment.

\section{Pre-processing treatment reads assembly and functional annotation}

Illumina Hiseq 2000 sequencing of 12 cDNA libraries (herein called samples) from four B. tabaci strains (3 biological replicates per strain) yielded more than 396 million paired-end reads. The pre-processing technique (low quality nucleotide reads trimming, removal of adaptors, low complexity sequences, poly $\mathrm{A} / \mathrm{T}$ tail) resulted to 369.04 million reads (72.75Gbp).

The short read sequences were deposited into the Sequence Read Archive (SRA), (BioProject ID: PRJNA293094). The full list of transcript sequences is available upon request from the corresponding author.

The number of reads was evenly distributed among the 12 libraries, with an average of $30.75+/-2.4$ million reads. The summary of reads per library used in the subsequent analysis is shown in Additional file 1: Table S1.

The intra- and inter-sample fraction for the contaminant ribosomal RNA as well as the mitochondrial cDNA was also similar among them $(5.3 \%+/-0.9,1 \%+/-0.4$ and $3.9 \%+/-0.70$ for total rRNA, hemiptera rRNA only and mitochondrial RNA, respectively) indicating moderate variation between libraries.

De novo assembly performed using Trinity produced 170,377 contigs (mean length: 1136 bp, N50: 2681 bp, Additional file 2: Table S2) corresponding to 113,450 unigenes (a set of contigs which are believed to belong together).

To evaluate the accuracy of the assembled sequences (transcripts), all the usable sequencing reads were aligned onto the transcripts using Bowtie2. A high percentage of reads (97.14\%) were successfully back- 
Table 1 The neonicotinoid response of the susceptible S-GR6 and the resistant B. tabaci laboratory strains. Toxicological data to the neonicotinoids imidacloprid, acetamiprid and thiacloprid and toxicity of imidacloprid after pre-exposure to synergist piperonyl butoxide $(\mathrm{PBO})$

\begin{tabular}{|c|c|c|c|c|}
\hline Strain & $n$ & LC50 (95 \% Cl) & RR $(95 \% \mathrm{Cl})$ & SR $(95 \% \mathrm{Cl})$ \\
\hline \multicolumn{5}{|l|}{ IMIDACLOPRID } \\
\hline S-GR6 & 580 & $7.5(5.4-10)$ & - & \\
\hline $\mathrm{S}-\mathrm{GR} 6+\mathrm{PBO}$ & 543 & $6.0(4.3-7.1)$ & - & - \\
\hline GR4_AP & 586 & $69(46-98)$ & $9(6-13)$ & \\
\hline GR4_AS & 479 & $423(258-657)$ & $56(36-87)$ & \\
\hline GR4_AS + PBO & 433 & $185(67-302)$ & $30(17-38)$ & $1.9(1.7-2.3)$ \\
\hline GR9_IP & 547 & $176(82-268)$ & $23(15-35)$ & \\
\hline GR9_IS & 479 & $301(186-443)$ & $40(28-58)$ & \\
\hline GR9_IS + PBO & 435 & $207(164-332)$ & $34(25-50)$ & $1.2(1.1-1.4)$ \\
\hline GRO_IP & 699 & $82(60-106)$ & $11(7-16)$ & \\
\hline GRO_IS & 451 & $177(55-336)$ & $24(14-39)$ & \\
\hline GRO_IS + PBO & 425 & $52(10-148)$ & $9(3-16)$ & $2.7(2.3-3.1$ \\
\hline \multicolumn{5}{|l|}{ ACETAMIPRID } \\
\hline S-GR6 & 503 & $7.9(5.1-11)$ & - & \\
\hline GR4_AP & 442 & $44(20-80)$ & $5(3-9)$ & \\
\hline GR4_AS & 539 & 157 (99-242) & $20(15-33)$ & \\
\hline GR9_IP & 391 & $56(28-89)$ & $7(4.1-11)$ & \\
\hline GR9_IS & 535 & $137(110-163)$ & $17(11-26)$ & \\
\hline GRO_IP & 402 & $33(25-59)$ & $4(2.8-6.3)$ & \\
\hline GRO_IS & 460 & $71(42-105)$ & $9(5.3-15)$ & \\
\hline \multicolumn{5}{|l|}{ THIACLOPRID } \\
\hline S-GR6 & 517 & $11(4.2-22)$ & - & \\
\hline GR4_AP & 422 & $>30,000$ & $>3,000$ & \\
\hline GR4_AS & 300 & $9,740(835-27,145)$ & $924(378-2,259)$ & \\
\hline GR9_IP & 449 & $>30,000$ & $>3,000$ & \\
\hline GR9_IS & 320 & $13,061 \quad(5,253-25,522)$ & $1,239(720-2,134)$ & \\
\hline GRO_IP & 422 & $>30,000$ & $>3,000$ & \\
\hline GRO_IS & 281 & $10,477(3,373-21,100)$ & $994(457-2,161)$ & \\
\hline
\end{tabular}

$R R$ resistance ratio at $L C 50$, the Lethal Concentration of $50 \%, C l$ confidence intervals resistance characteristics, $S R$ synergist ratio: RR/RR after pretreatment with PBO

mapped on this assembly and at least $71.6 \%$ of the mapped reads were proper pairs.

Similarity between the Trinity assembly and $n r$ NCBI protein database was examined using Blastx and disclosed 31,505 contigs with coverage higher than $80 \%$. In addition, similarity to a dataset of 129 complete mRNA of $B$. tabaci downloaded from NCBI, using blastn, disclosed 134 contigs and $106 \mathrm{~B}$. tabaci mRNA sequences that met the criteria initially set (>95\% identity, coverage of the contig or subject $90 \%$ [31]). The average identity was $98.37 \%$, similar to the level of sequencing accuracy already reported for Illumina technology [31].

The blastx search (e-value cut-off $<10^{-6}$ ) returned 37,393 contigs with at least one blast hit. Additionally, 1,988 contigs were found with blastn performed on the remaining (with no blastx hit) contigs (e-value cut-off $<10^{-10}$ ) (Table 2). InterPro Scan returned 67,687 contigs with a protein domain match.

To identify the possible functions, Gene Ontology (GO) assignments were used to classify the sequences. A total of 25,392 contigs with at least one GO term (Table 2) were found. The sequences were categorized to ten molecular function (MF), thirteen biological process (BP) and seven cellular unigenes (CC) categories in GO level 2 (general function categories) (Additional file 3: Figure S1). The GO classification results are in line with previous sequenced transcriptomes of B. tabaci [15, 32], T. vaporariorum [19] and $B$. oleae [33] where binding, cell, catalytic activity and metabolic process were the four largest groups. 
Table 2 Annotation summary

\begin{tabular}{llclc}
\hline & Contigs & \% contigs & Unigenes & \% unigenes \\
\hline & 170,377 & & 113,450 & \\
BLAST & 39,381 & 23.11 & 16,126 & 14.21 \\
InterPro & 67,687 & 39.73 & 38,011 & 33.50 \\
With IPR number & 32,945 & 19.34 & 12,866 & 11.34 \\
With > =1GO & 25,270 & 14.83 & 10,104 & 8.91 \\
Blast2Go annotated & 25,392 & 14.90 & 10,685 & 9.42 \\
EC & 6,994 & 4.02 & 3,230 & 2.85 \\
KEGG 137 pathways & 6,731 & 3.95 & $1,513^{\mathrm{a}}$ & 1.33 \\
\hline aUnique unigenes & & & &
\end{tabular}

The potential enzymes were characterized based on the chemical reaction they catalyze using the prediction of Enzyme Commission (EC) numbers for each sequence. Among the 37,393 annotated contigs, 6,994 contigs were classified to 795 different EC numbers. Enzyme classification revealed that transferases are the largest group of $B$. tabaci MED enzymes (34 \%, 269 enzymes) followed by oxydoreductases (22\%, 178 enzymes), hydrolases (22\%, 176 enzymes), lyases (10 \%, 76 enzymes) ligases (8 \%, 65 enzymes) and isomerases (4\%, 31 enzymes).

To identify the biological pathways that are active in the $B$. tabaci MED, we mapped all contigs with EC number (3,230 unigenes) to the reference canonical pathways in Kyoto Encyclopedia of Gene and Genome (KEGG). In total, we assigned 6,731 (out of 6,994) contigs to 137 KEGG pathways. The pathways mostly represented by the assembled sequences were purine metabolism (52 enzymes, 624 contigs), arginine and proline metabolism (29 enzymes, 95 contigs) and pyrimidine metabolism (28 enzymes, 174 contigs). Contigs for pathways that could be involved in detoxification like metabolism of xenobiotics by cytochrome P450 (7 enzymes, 59 contigs), drug metabolism - cytochrome P450 (6 enzymes, 51 contigs), drug metabolism - other enzymes (15 enzymes, 77 contigs), oxidative phosphorylation (7 enzymes, 113 contigs), glutathione metabolism (15 enzymes, 88 contigs) and nicotinate and nicotinamide metabolism (11 enzymes, 78 contigs) were also represented (Additional file 4: Table S3).

Sequences encoding the targets of the major classes of synthetic insecticides as well as enzymes potentially involved in the xenobiotic detoxification were extracted and compared with sequences from the NCBI protein database. Genes putatively involved in the insecticide metabolic resistance are summarized in Table 3, including conventional detoxification enzymes such as cytochrome P450 monooxygenases (P450s, 146 unigenes), carboxylesterases (CCEs, 23 unigenes) glutathione S-transferases (GSTs, 18 unigenes), UDP-glycosyltranferases (UGTs, 35 unigenes) and $\mathrm{ABC}$ transporters (35 unigenes). Putative insecticide targets including nicotinic acetylcholine receptor ( $\mathrm{AAChR}, 10$ unigenes), gamma-aminobutyric acid receptor (GABA, 7 unigenes) and acetylcholinesterase (AChE, 3 unigenes) are also shown in Table 3.

\section{Investigating target-nicotinic acetylcholine receptors- mediated resistance}

In other insect species, mutations as well as alterations in the expression levels of nAChRs, the target of neonicotinoids, have been associated with neonicotinoid resistance [9]. Focused on these genes, we performed a phylogenetic study and investigated sequence polymorphism and expression levels of the nAChRs.

Nicotinic acetylcholine receptors (nAChR) are ligand gated ion channels (LGIC) and serve an important role in nerve signaling, at the post-synaptic membrane, by mediating the fast actions of acetylcholine (ACh) at cholinergic synapses. Neonicotinoid insecticides and spinosad have high and selective affinity for many arthropod nAChRs [34].

The phylogenetic analysis including 25 contigs and 97 $\mathrm{nAChR}$ protein sequences from other insect species revealed $10 \mathrm{nAChR}$ orthologs in B. tabaci MED encoding putative receptor subunits (Fig. 1, and for alignment Additional file 5: Figure S2). A comparative number of subunits (10 and $12 \mathrm{nAChR}$ subunits) was found in $A$. pisum, An. gambiae, Ap. mellifera, B. mori, D. melanogaster and $T$. castaneum. Nine nAChR subunits, $\alpha 1, \alpha 2$, $\alpha 3, \alpha 4, \alpha 5, \alpha 6, \alpha 7, \alpha 8$, and $\beta 1$ were grouped into highly conserved core groups and were named accordingly [35]. Interestingly, as in other insects, B. tabaci contained a subunit which showed particularly low sequence identity with other $\mathrm{nACh}$ receptors and was clustered into a divergent group including subunits $\alpha 9$ and $\beta 2$ of A. mellifera, $\beta 3$ of D. melanogaster, $\alpha 9$ and $\alpha 10$ of T. castaneum. The bidirectional best hits of this subunit against all insects and acari $\mathrm{nAChR}$ are for Locusta migratoria $\alpha 9$ (46\% similarity) and T. castaneum $\alpha 10$ (44\%). However, in this subunit one of the two vicinal cysteines of the motif YxCC at Loop C, defining $\alpha$ subunits, was replaced by a histidine and is therefore named $\beta 2$ (Additional file 6: Figure S3).

Sequence comparison results show that most of the $B$. tabaci nAChR subunits contained a GEK motif preceding TM2 (Additional file 6: Figure S3), a highly conserved motif in most insect $\mathrm{nAChR}$ subunits where the glutamate residue is considered to be associated with cation selectivity. However, the GEK motif was replaced by REK in $\alpha 2$ and by MER in $\beta 2$. Such results have been also observed in $B$. mori where the motif was replaced by STR in $\alpha 9$ and $\beta 3$, and by TIR in $\beta 2$ [36]. Whether $B$. tabaci MED $\alpha 2$ and $\beta 2$ possess the cation selectivity as other GEK-motif-containing AChR subunits remains to be determined. 
Table 3 Summary of contigs putatively involved in insecticide resistance

\begin{tabular}{|c|c|c|c|c|c|}
\hline Family & Contigs & Unigenes & Hits & Average contig length (bp) & Average aminoacid length (bp) \\
\hline \multicolumn{6}{|l|}{ Detoxification } \\
\hline $\mathrm{ABC}$ transporter & 82 & 35 & 36 & $3,557.9$ & 689.3 \\
\hline CCE & 59 & 23 & 11 & $1,816.8$ & 419.0 \\
\hline CPR & 2 & 1 & 1 & $4,018.5$ & 694.0 \\
\hline Esterase & 152 & 51 & 58 & $3,741.2$ & 537.6 \\
\hline Glutathione peroxidase & 1 & 1 & 1 & $2,100.0$ & 204.0 \\
\hline GST & 52 & 18 & 19 & $1,512.9$ & 232.7 \\
\hline Hydrolase & 136 & 68 & 71 & $3,242.4$ & 490.6 \\
\hline $\mathrm{P} 450^{\mathrm{a}}$ & 367 & 146 & 91 & $1,764.6$ & 364.1 \\
\hline Peroxidase & 13 & 8 & 8 & $4,125.4$ & 701.0 \\
\hline UGT & 69 & 35 & 22 & $1,791.5$ & 377.7 \\
\hline \multicolumn{6}{|l|}{ Target } \\
\hline ACCase & 1 & 1 & 1 & $5,633.0$ & $1,751.0$ \\
\hline AChE & 5 & 3 & 3 & $2,603.8$ & 500.4 \\
\hline Chitin synthase & 1 & 1 & 1 & $5,600.0$ & $1,571.0$ \\
\hline Chloride channels & 58 & 10 & 15 & $3,037.4$ & 527.5 \\
\hline GABA & 17 & 7 & 7 & $1,971.9$ & 431.1 \\
\hline GluCl & 12 & 2 & 3 & $1,676.2$ & 383.2 \\
\hline$n A C h R^{b}$ & 28 & 10 & 10 & $1,872.9$ & 414.1 \\
\hline Ryanodine receptor & 1 & 2 & 1 & $15,901.0$ & $3,833.0$ \\
\hline Sodium channel & 41 & 12 & 11 & $2,303.8$ & 624.7 \\
\hline \multicolumn{6}{|l|}{ Other } \\
\hline Cuticular protein & 32 & 17 & 16 & $1,470.6$ & 272.6 \\
\hline Cytochrome b5 reductase & 3 & 3 & 3 & $2,377.7$ & 405.3 \\
\hline Catalase & 7 & 4 & 5 & $2,672.1$ & 452.2 \\
\hline Superoxide dismutase & 11 & 5 & 6 & $1,702.0$ & 208.9 \\
\hline NADH dehydrogenase & 38 & 34 & 33 & $1,358.3$ & 136.1 \\
\hline NADH oxidoreductase & 4 & 4 & 4 & $1,680.8$ & 298.3 \\
\hline
\end{tabular}

First blast hit, E-value cut-off $<10 \mathrm{E}-10$

${ }^{a}$ First tblastn hit against insect $\mathrm{P} 450$ proteins

${ }^{b}$ First tblastn hit against insect $n A C h R$ proteins

It is worth noting that two subunits were represented by variant isoforms: $\alpha 4$ and $\alpha 5$ with 2 and 3 variants respectively. Alternative splicing occurs commonly and has been observed in the $\alpha 3, \alpha 4, \alpha 6, \alpha 7$ subunits in many insects including A. melifera [37], A. pisum [38], B. mori [36], T. castaneum [39]. It effectively diversifies receptor function and generates species-specific isoforms by introducing variations in the Cys-loop that are involved in receptor assembly, in loops $\mathrm{E}$ and $\mathrm{B}$. These variations contribute to ligand binding in the second transmembrane domain, which forms the ion channel between TM2 and TM3, that are important in coupling agonist binding to ion channel gating $[40,41]$. Together with alternative splicing, post transcriptional RNA A to I editing (especially in $\alpha 6$ subunit) and other alterations like truncated transcripts ( $\alpha 7$ and $\alpha 4)$ contribute in largely increase the diversification of $\mathrm{nACh}$ receptors between insect species [35, 39, 42-44] and counterpart the low number of insect subunits compared to vertebrate nAChR. Whether the different isoforms observed in nAChR subunits $\alpha 4$ and $\alpha 5$ of $B$. tabaci consist of alternative transcripts or not, clearly warrant further investigation by combining transcriptomic and genomic data.

We found substantial variation in expression levels between the different subunits of $\mathrm{nACh}$ receptors. In all strains, subunit $\beta 2$ was the most highly expressed (average FPKM across libraries $=52$ to 100 ) followed by the $\alpha 2(\mathrm{FPKM}=6$ to 8$)$ and the similarly expressed $\alpha 3, \alpha 7$ and $\alpha 8($ FPKM $=3$ to 6$)$. The lower expressed subunit was $\alpha 6$ with only ten mapped reads (Fig. 2). We also examined if differential gene expression occurred between strains. DE was found for $\beta 2$ between S-GR6 and GR4- 

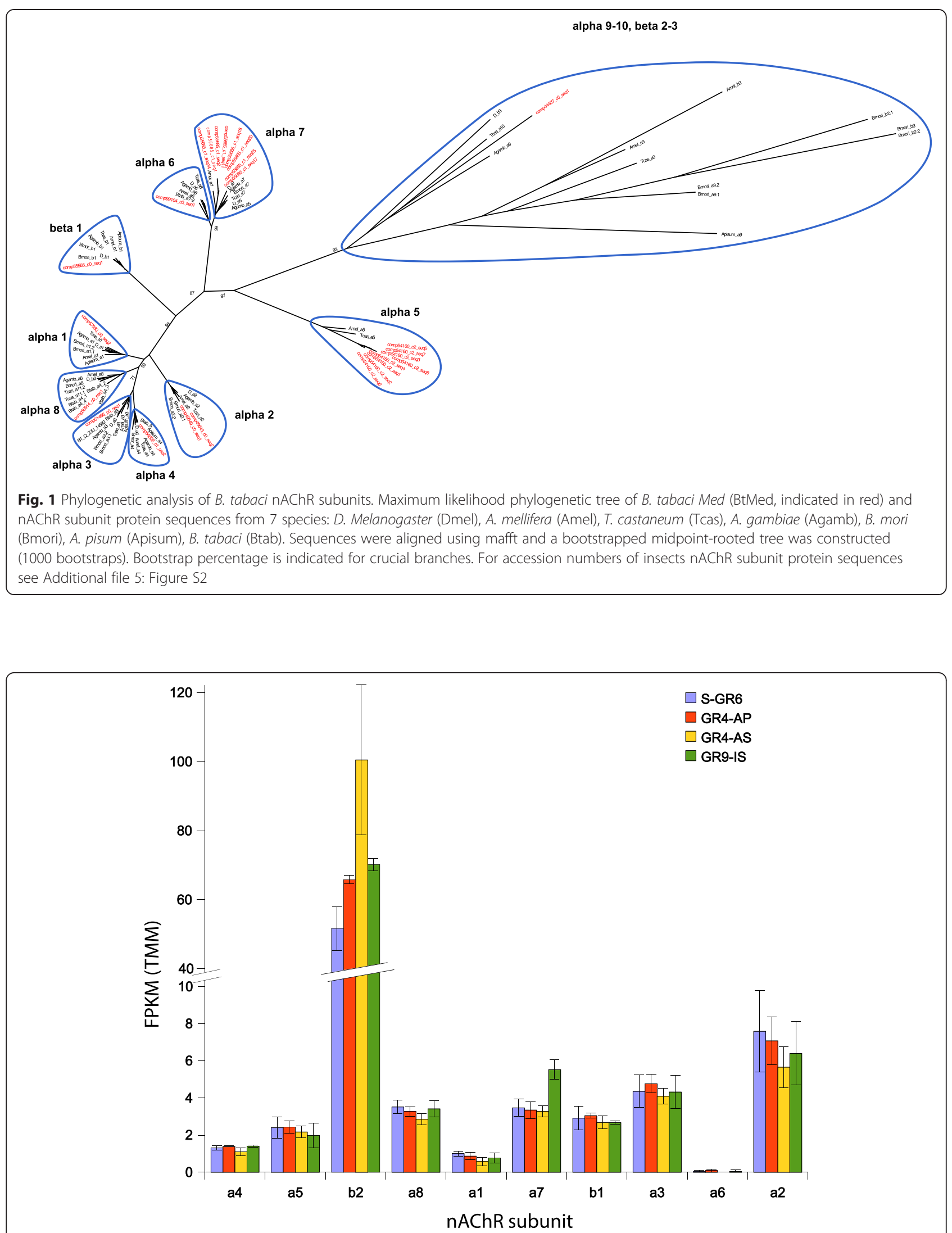

Fig. 2 Graph bar diagram of FPKM normalized (TMM) expression level of B. tabaci nAChR subunits 
AP $\left(\operatorname{logFC}=0.94\right.$, FDR $\left.=9.210^{-5}\right)$ or GR4-AS $(\operatorname{logFC}=$ $0.60, F D R=0.032$ ) and for subunit $\alpha 7$ between S-GR6 and GR9-IS $(\operatorname{logFC}=0.65, F D R=0.011)$. Whether this differential gene expression between resistant and susceptible strains for subunits $\beta 2$ and $\alpha 7$ contributes to the observed resistance phenotypes needs further examination. However, it is interesting to point to recent studies that established a link between nAChR expression and response to neonicotinoids. Taillebois et al. [45] showed that pre-exposure to different neonicotinoids significantly affected the expression levels of some subunits which were depended on the nature of the insecticide. The importance of differential expression of subunits in the regulation of neonicotinoid sensitivity is also suggested by the findings of $\mathrm{Yu}$ et al. [46], who observed a significant decrease of expression of $\beta 1$ and $\beta 2$ after exposure to imidacloprid. Moreover, Markussen and Kristensen [47] found that neonicotinoid resistance phenotype in Danish Musca domestica was correlated with reduced expression of the nAChR subunit $\alpha 2$.

We investigated the occurrence of SNPs in unigenes encoding $\mathrm{nAChRs}$ and possible differences between resistant and susceptible B. tabaci strains. A total of 347 SNPs were identified and were equally distributed among strains (283 in S-GR6, 324 in GR9-IS, 297 in GR4-AS and 310 in GR4-AP). Most of them (52\%) were found in the coding regions, $19 \%$ in the 5 'UTR and $29 \%$ in the 3 'UTR.

The number of SNPs differed between the subunits of nAChR with the subunits $\alpha 8$ and $\beta 1$ showing higher variation (111 and 68 SNPs, respectively) and subunits $\alpha 5$ and $\alpha 7$ lower variation (only 1 and 2 SNPs, respectively). In subunit $\alpha 6$, no variable site was detected; however, the shorter transcript was recovered for this subunit.

Comparative polymorphism analysis identified several alleles differentially represented between insecticide resistance strains and the reference susceptible strain ( $>50 \%$ allele frequency difference, referred to as 'differential SNPs'). Twenty-seven out of the 347 SNPs were differential SNPs (10 in the coding region and 17 in the 5'UTR or in the 3'UTR). Seventeen polymorphisms concerned SNPs with high frequency differences between the S-GR6 and any of the resistant strains, which were equally affected $(15,15$ and 16 differential SNPs for GR9-IS, GR4-AP and GR4-AS, respectively). Only five differential SNPs between $S$ and $R$ strains were in the coding region and all of them concerned synonymous substitutions.

Among all transcripts, five out of the 347 SNPs annotated in the $\mathrm{nAChR}$ sequence assembly were found in subunits $\beta 2$ (3), $\alpha 3$ and $\alpha 4$, resulted in an amino acid change (Additional file 6: Figure S3). In subunit $\beta 2$, one non-synonymous SNP (nsSNP) was found at the $\mathrm{N}$ terminal signal peptide and involved an alteration of $\mathrm{F}$ and $\mathrm{S}$ residue at position 12 (aa numbering as in alignment of Additional file 6: Figure S3) and two others, D/E and N/T at positions 400 and 426, were found between transmembrane domain TM3 and TM4. In subunit $\alpha 3$, a nsSNP (P/ A) was found at position 448 between TM3 and TM4. Finally, an F/Y change has been detected in subunit $\alpha 4$ located at 159 residues between LpA and LpE. None of the mutations leading to substitutions known to confer target resistance to neonicotinoids in other insect pests were detected (coverage 65 to 309 for the specific sites). Despite the apparent scarcity of target site resistance to neonicotinoids compared to increased detoxification, two target mutations conferring resistance to neonicotinoids have been documented in other agricultural pests $[9,48]$. An amino acid substitution in a highly conserved site of the loop B of two nAChR subunits ( $\alpha 1$ and $\alpha 3$ ), that causes a tyrosine to serine substitution (Y151S), was detected in the brown planthopper Nilaparvata lugens conferring imidacloprid resistance [9]. The apparent scarcity of this mutation, in any other insect species including neonicotinoid resistant $B$. tabaci, could be explained (as discussed by Liu et al. [9]) by the hypothesis that insects may need two mutated subunits to express resistance. Another mutation in a highly conserved site within loop $\mathrm{D}$ of $\beta 1$, that causes an arginine to threonine substitution (R81T), was found in highly resistant clones of the aphid Myzus persicae [48]. In addition, the substitutions found in our study were not located in any of the hot spot regions of the $n A C h R$ subunits i.e. loops $D, E$ and $F$ of subunit $\beta 1$, or loops $A, B$ and $C$ of $\alpha$ subunits. These regions form the binding site for acetylcholine and for certain agonists including neonicotinoids [49], where a number of key residues for toxin and neonicotinoid binding and selective toxicity in insect $\mathrm{nAChR}$ have been identified by functional expression of hybrid or chimeric receptors and by docking simulations (reviewed in $[35,50]$ ).

\section{Investigation of non - target site resistance mechanisms based on differential expression}

We compared gene expressions of the susceptible and the three neonicotinoid resistant strains and performed differential expression analysis (DE). Data of three biological replicates of each condition were used in this analysis (in total twelve samples). The biological coefficients of variation $(\mathrm{BCV})$ observed in this study ranged from 0.11 to 0.19 (BCV: GR4-AS = 0.19, GR4-AP = 0.11, GR9-IS $=0.15, \mathrm{~S}-\mathrm{GR} 6=0.17$, mean $0.16 \mathrm{SE}=0.018$ ) and are close to those observed in genetically identical model organisms, indicating small variability between replicates [51]. The low intra biological replicate variation and the clear separation between susceptible and the three resistant strains provided a solid base to all subsequent analyses (Additional file 7: Figure S4). 
The DE analysis revealed in total 3,745 differentially expressed unigenes $(|\log \mathrm{FC}|>1, \mathrm{FDR}<0.05)$ in the three $\mathrm{R}$ strains compared to the $\mathrm{S}$ strain based on their TMMFPKM (Additional file 8: Table S4). According to the expression levels of the 3,745 DE unigenes we examined the overall differential expression and detected three sub-clusters that exhibited marked differences in normalized FPKM. This clustering grouped the neonicotinoid resistant samples together. Assigning known transcripts to molecular functions revealed differences in the GO enrichment in the three clusters, showing that substantial differences in the transcriptional profile between resistant and susceptible strains exist (Additional file 9: Figure S5).

The frequency of the up- and down-regulated unigenes was not significantly different within each strain. Differential transcription was homogenously distributed between up- and down-regulated unigenes in all strains with an almost $50 \%$ frequency: 1,077, 1,250 and 847 unigenes were up regulated and 928, 1,132 and 794 unigenes down regulated in GR9-IS, GR4-AS and the GR4AP, respectively, compared to the susceptible S-GR6 strain $(|\log \mathrm{FC}|>1, \mathrm{FDR}<0.05)$ (Fig. 3a).

The total number of DE unigenes against S-GR6 was unbalanced (Fig. 3) suggesting a quantitative difference in transcription response between strains, while the lower number of DE unigenes was found in GR4-AP. A total of 1,539 unigenes were found differentially expressed in any strain, including 332 and 405 unigenes over and under transcribed in all strains, respectively. The two selected strains (GR4-AS and GR9-IS) showed the higher number of DE unigenes with 584 and 528 transcripts specifically over-transcribed as compared to the susceptible strain (Fig. 3b). In contrast, fewer transcripts were differentially expressed in the parental strain (GR4-AP), maintained with no selection, with only 212 specifically over transcribed genes. Not surprisingly, the comparison between GR4-AS versus GR4-AP revealed the lower number of regulated genes, 159 up- and 167 down-regulated unigenes (Fig. 3b).

For a better understanding of the molecular functions and biological processes underlying the nature of resistance to insecticides, Gene Ontology term enrichment analysis was used to detect differentially expressed unigenes over or under represented specifically in each strain. Here, the term enrichment results for the comparison between the DE unigenes of each strain against the reference transcriptome. For the up-regulated unigenes, the GO terms were generally negatively enriched in the resistant strains including terms such as response to stimulus (GO:0050896), signalling (GO:0023052) and cellular process (GO:0009987) in all three resistant strains and terms such as binding (GO:0005488) only in GR4_AS. Among the higher significant positively enriched GO terms corresponding to up-regulated unigenes figured those related to catalytic activity such as transferase (GO:0016740) and oxidoreductase activity (GO:0016491) in all three resistant strains together with terms related to heterocyclic compound binding (GO:1901363) and organic cyclic compound binding (GO:0097159) in GR4_AP and GR9_IS (Additional file 10: Table S5, Fig. 4).

In genes with up-regulation in the GR4_AS compared to its parental GR4_AP hydrolase (GO:0016787) and peptidase activity (GO:0008233) are among the terms related with catalytic activity (GO:0003824), for which the frequency increased significantly (Fig. 4). Interestingly, among the down-regulated unigenes the term "response to stress" (GO:0006950) figures among the over represented terms suggesting that genes related to stress are not only down-regulated in GR4-AS compared to the parental but also represent a higher percentage of total GO terms down regulated compared to the reference transcriptome (Additional file 10: Table S5).

In addition to the differential gene expression of nAChR subunits between resistant and susceptible strains that was examined in the "target" paragraph, the expression profiles of other genes known to be involved in insecticide resistance were closely examined (logFC $>1$, FDR $<0.05$ ) (Additional file 8: Table S4). This DE analyses includes genes related to insecticide detoxification or altered insecticide transport and sequestration.

Among the 550 differentially over-transcribed unigenes in any $\mathrm{R}$ strain with a Blast hit, several genes related to altered insecticide transport and sequestration were found including five cuticular proteins (one unigene with $\operatorname{logFC} 2.48$ and 4.27 in $\mathrm{AS}$ and AP, respectively; GO:0042302) and five fatty acid synthases (Additional file 8: Table S4). Typically, the metabolic detoxification system in insects consists of enzymes, acting on a broad range of substrates directly to reduce their toxicity, represented by cytochrome P450 monooxygenases (P450s), and carboxylesterases (CCEs) or to facilitate the excretion of hydrophobic toxic compounds by improving their hydrophilicity such as glutathione-S-transferases (GSTs) and UDP-glucuronosyltransferases (UGTs). The strong response of the GR9-IS, GR4-AS and GR4-AP to insecticide selection through transcription level modifications was confirmed. Among the 550 differentially over expressed unigenes in any $\mathrm{R}$ strain with a Blast hit, several detoxification enzymes were represented including unigenes with orthologs in P450s (35 unigenes), GSTs (2), CCEs (8) and UDP-glucuronosyltransferases (7) with some of them, namely $\mathrm{P} 450 \mathrm{~s}$, displaying a $\log \mathrm{FC}>5$ (Additional file 11 : Table S6).

The between strains comparison displayed that, compared to the susceptible strain S-GR6, the number of the up-regulated DE detoxification unigenes (36, 32 and 18 


\section{a}

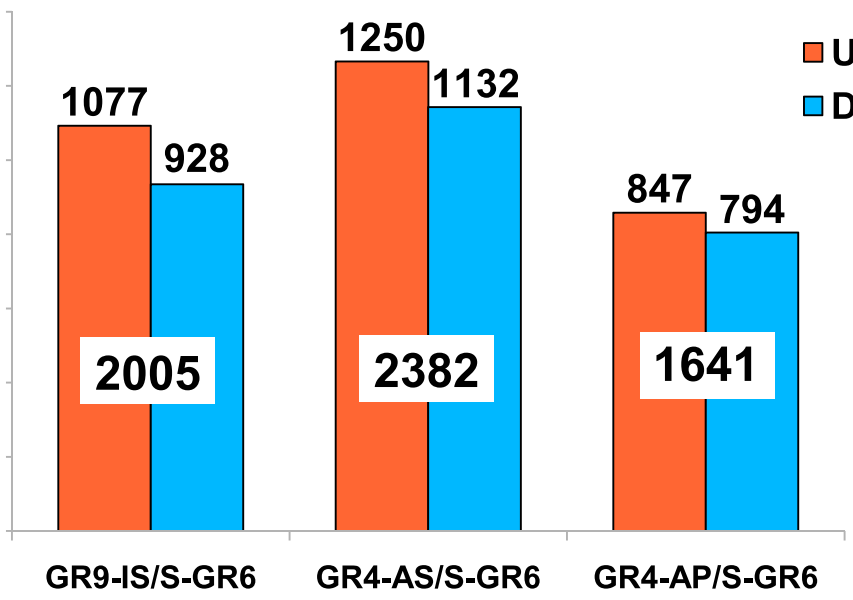

b

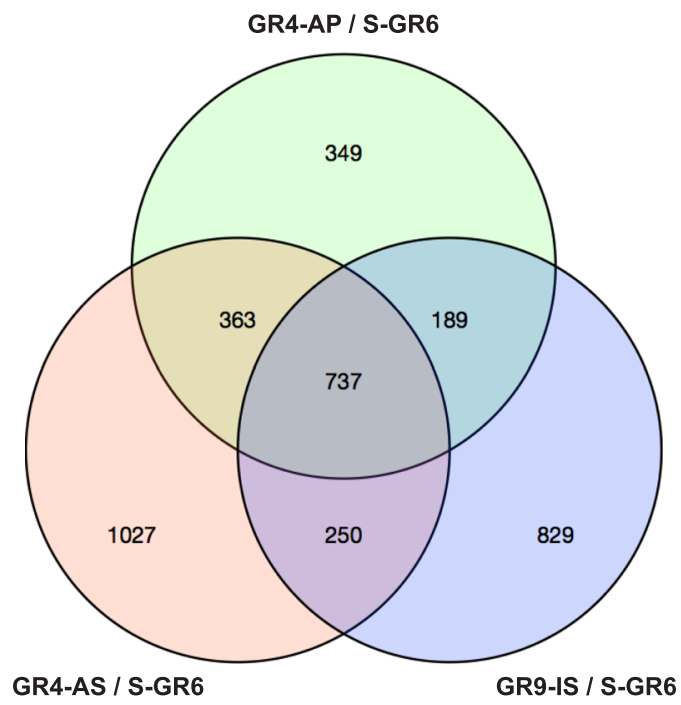

Fig. 3 Graph bars and venn diagram of differentially expressed unigenes of the three resistant strains. a) Distribution of differentially expressed unigenes of the resistant strains compared to the susceptible strain $(\log 2 F C>1, F D R<0.05)$. In each strain up and down regulated unigenes are indicated. b) Venn diagram of differentially expressed unigenes in the three resistant strains. For each Venn diagram section, the numbers of transcripts differentially expressed in any insecticide resistant strain as compared to the susceptible strain are indicated

in GR4-AS, GR4-IS and GR4-AP, respectively; Additional file 11: Table S6) differed significantly (Fisher exact test) between the GR4-AP and either GR4-AS or GR9-IS, but not between GR9-IS and GR4-AS. Moreover, this number is in accordance with the resistance ratios observed in the three neonicotinoid resistant strains (Table 1) suggesting a quantitatively differentiated transcriptional profile related to insecticide detoxification.

GR4-AP shared all of its up regulated detox unigenes with at least one of the selected strains. The 13 unigenes shared by all resistant strains (including the parental relaxed strain) should represent genes selected by neonicotinoid or other insecticides applied in the field and maintained their expression levels in GR4-AP strain even after 6 generations without insecticide exposure. The four significantly up regulated unigenes shared only by the GR9-IS and GR4-AS, which may have been commonly selected by either neonicotinoids, include three $\mathrm{P} 450$ s and one CCE with moderate $\operatorname{logFC}$ ranging from 1 to 2 (Additional file 11: Table S6).

Investigation of detoxification genes specifically overexpressed in each resistant strain disclosed that two CCEs, one UGT, and 11 P450s (in total 14) were specifically up regulated in GR9-IS strain, with one CCE and one P450 having the higher $\operatorname{logFC}$ (2.7 and 3.7 respectively). Similarly, 17 unigenes were specifically up regulated in GR4-AS including 10 P450, 4 UGTs, 2 GSTs 


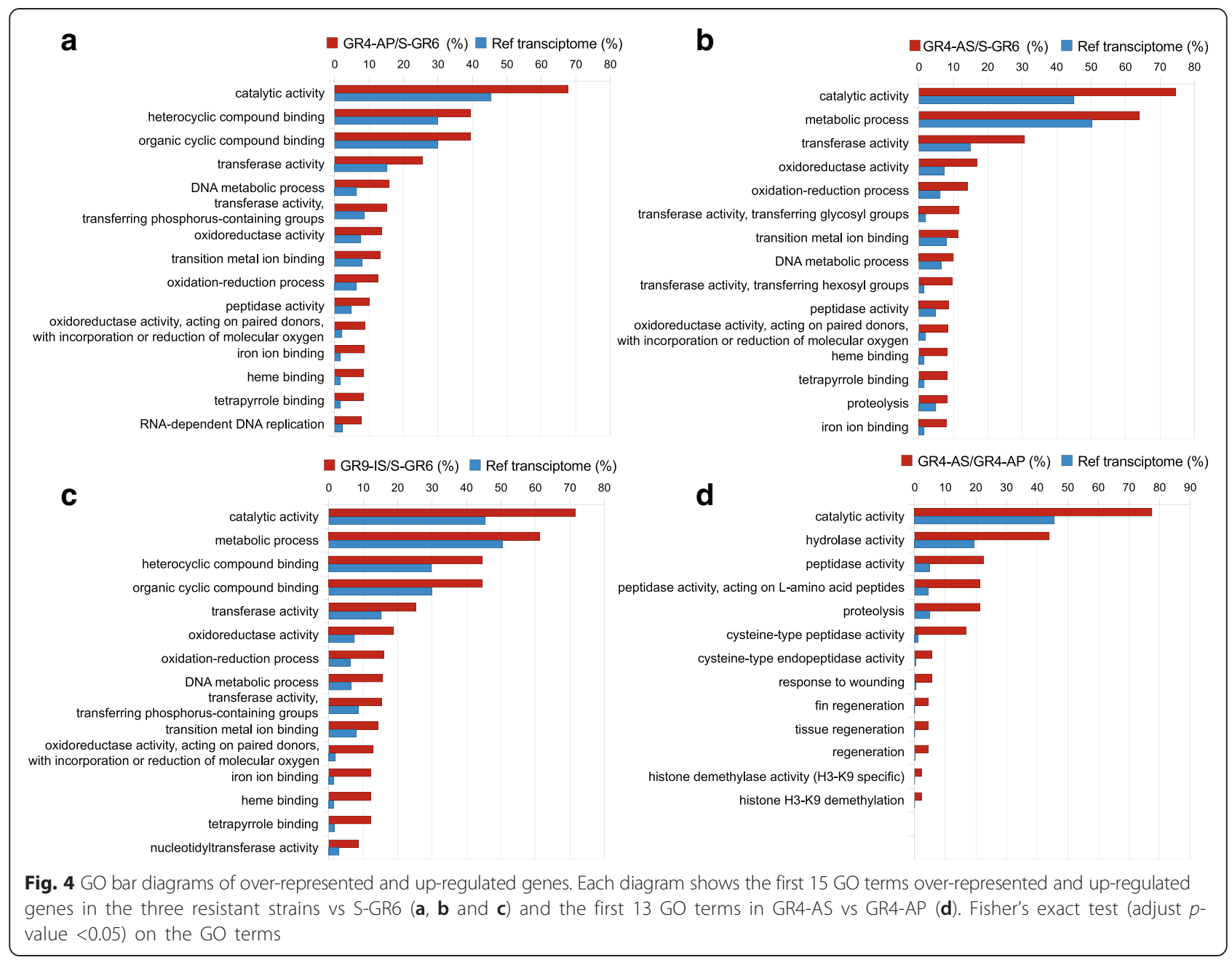

and 1 CCE. These specifically GR9-IS or GR4-AS up regulated unigenes reflect a genetic background related specificity, a selection agent (imidacloprid or acetamiprid) specificity, or a combination of both. Our experimental design does not allow distinguishing between the two cases. However, taking into account that these strains should belong to the same genetic cluster (south Crete) identified by microsatellites [52], it is possible that the different (specific) up-regulated unigenes in each GR9-IS or GR4-AS strain reflect insecticide specificity. One P450 unigene (comp43065_c0) was upregulated in the GR4-AS compared to both S-GR6 and its parental strain GR4-AP $(\log F C>2.5)$. This unigene might represent a gene with a positive transcription reaction to the selection with acetamiprid and should be studied further.

GSTs metabolism as a mechanism of resistance to organochlorines, carbamates, organophosphates and pyrethroids has been reported in many arthropod species [5356]. Similarly enhanced production of carboxylesterases through gene amplification and/or up-regulation has been demonstrated in several organophosphate, carbamate or pyrethroids resistant insects [57-59]. However, GSTs or COE have never been directly associated with neonicotinoid resistance and therefore the over-expression of the GSTs and COEs in the GR9_IS, GR4_AP and GR4_AS may have been selected as a result of contact with other xenobiotics in their environment.

Among detoxification enzyme systems, P450s are the most common genes found up-regulated in a wide number of resistant insects with a subset of these P450s confirmed to be able to metabolize pesticides by functional in vitro studies in mosquitoes species reviewed by $[60$, 61], $M$. domestica [62], D. melanogaster [63], N. lugens [64], H. armigera [65, 66], T. urticae $[67,68]$ and $B$. tabaci $[10,69,70]$. In addition, monooxygenase-mediated neonicotinoid resistance has been reported in several insect species including $B$. tabaci $[11,21,29,71,72], M$. domestica [73], M. persicae [74] and D. melanogaster [75]. P450s expression profile is examined in more details in the following paragraphs. 


\section{Detailed study of transcripts encoding putative P450s} Phylogenetic study of transcripts encoding putative P450s

P450s are one of the largest super-families, playing a dominant role in plant-insect interactions and insecticide/xenobiotic metabolism. They are divided in 4 major clades, named CYP2, CYP3, CYP4 and mitochondrial [30]. A variable number of $\mathrm{P} 450$ s has been identified in insect genomes such as D. melanogaster (88), A. gambiae (105), A. aegypti (160), T. castaneum (134), A. pisum (64) (reviewed in [30]) and in recent transcriptome studies of B. dorsalis (90), B. oleae (55) and $T$. vaporariorum (57) [19, 31, 33].

A total of 367 contigs (146 unigenes) were identified as $\mathrm{P} 450$ encoding genes in the B. tabaci transcriptome (Additional file 12: Table S7). To assign B. tabaci P450s to respective P450 clusters and families, a phylogenetic analysis was performed and, in the case of misaligning protein sequences, the closest blastp hits in the NCBI $n r$ database were identified. In the phylogenetic analyses, only the longest 171 non-redundant contigs (115 unigenes) were included together with other known insect P450s (345 protein sequences from 14 species including B. tabaci).

In this dataset, representatives of all 4 major insect P450 clades were found. The majority of B. tabaci P450s (76 out of 171) belonged to the CYP3 clade, 73 to the CYP4, 18 to the CYP2, and 4 to the mitochondrial clade (Additional file 13: Figure S6). Most of B. tabaci P450s included in CYP3 and CYP4 clades cluster separately from P450s from other insect species of the phylogenetic analysis. In CYP4, the separate clusters formed by $B$. tabaci $\mathrm{P} 450$ s are supported by high bootstrap values and most of them form sister groups to other Hemiptera P450s (ex A. pisum). However, this separation is not as clear in CYP3 B. tabaci P450s.

Finally, we performed a Bidirectional Best Hit (BBH) with 1:1 relationship between the $171 \mathrm{P} 450$ s contigs of the current study and the $345 \mathrm{P} 450$ proteins from different species that were also included in the phylogenetic analysis. The $\mathrm{BBH}$ returned $35 \mathrm{P} 450$ orthologs. For the remaining $\mathrm{P} 450 \mathrm{~s}$, a corresponding name was assigned according to the first Blast hit. (Additional file 12: Table S7).

\section{Expression levels of transcripts encoding putative P450s}

The phylogenetic analyses showed that most of the 35 up-regulated P450 unigenes in any B. tabaci strain belonged to the two larger P450 clades CYP4 and CYP3, with 18 and 15 unigenes, respectively. Three unigenes belonged to CYP2 and only one in the mitochondrial clade. More specifically, most of them corresponded to genes from CYP6, CYP4 and CYP417 families, but also from CYP303, CYP301, CYP304 and CYP439 families (Additional file 12: Table S7). We subsequently focused on the higher over-expressed P450 unigenes which displayed $\log \mathrm{FC}>2$ in at least one resistant strain. Eight unigenes that belonged to clades CYP2 (2), CYP3 (3) and CYP4 (3) met this criterion. Six unigenes (comp50040_c0, comp61334_c0 comp62212_c0, comp57969_c113, comp57969_c124 and comp33028_c0) were up regulated in all resistant strains compared to the S-GR6 strain. One unigene, comp43434_c1, was highly up-regulated only in GR9_IS $(\operatorname{logFC}=3.7)$ and another, comp43065_c0, only in the GR4_AS strain $(\operatorname{logFC}=5.4$ ) (Additional file 12: Table S7). In all cases, P450 expression was higher in the GR4_AS maintained under acetamiprid selection, compared to its corresponding parental relaxed GR4_AP suggesting the possible contribution in acetamiprid metabolism. However, a significant difference between selected and parental strains has been found only in the comp43065_c0 (Additional file 12: Table S7).

Unigenes comp61334_c0 and comp33028_c0 shared the higher similarity (higher best hit) with a putative P450 CYP303a1-like of A. pisum and in the phylogenetic analysis clustered together with CYP303a1 of A. pisum and $L$. striatella (100 \% bootstrap). They were both significantly up-regulated in the resistant strains compared to the S-GR6 however, comp61334_c0 displayed higher up regulation $(\operatorname{logFC}=5.57-6.58)$ compared to comp33028_c0 $(\log \mathrm{FC}=1.4-3.2)$. These two specific P450s belong to clade CYP2 and are presumed 1:1 orthologs with CYP303a1 family members. Interestingly, CYP303A 1 is one of few highly conserved P450s found in all insect species sequenced to date, but not in Daphnia pulex [30]. To our knowledge members of this family have never been associated with resistance to any insecticide and this is the first report for such a finding. In D. Melanogaster, CYP303A1 has a putative external sensory development function and it has been hypothesized that it probably metabolizes a small signal molecule essential in the development and structure of external sensory organs $[30,76]$. Whether this has to do with the sensorial capacity and avoidance of treated surface behavior remains unknown.

Components 62212_c0 and 43434_c1 shared the higher similarity with CYP417B1 of L. stratiella (orthologous to CYP417). They belong to the CYP4 clade, with which they formed a separate cluster with other P450 unigenes of $B$. tabaci issued from this study. This cluster was a sister group with members of CYP417, CYP425, CYP426 and CYP439 of L. stratiella. Comp62212_c0 was highly up-regulated in the three resistant strains $(\log \mathrm{FC}=4.78-6.07) \quad$ while comp43434_c1 was upregulated only in the GR9-IS strain $(\operatorname{logFC}=3.73)$.

A third unigene of clade CYP4, comp43065_c0, shared the higher similarity and was 1:1 ortholog with CYP4C1 of $A$. pisum, with which formed a distinct group separated from other CYP4C1 A. pisum sequences. This unigene was highly over-expressed in the GR4-AS strain 
$(\log \mathrm{FC}=5.36)$ and was also significantly increased level of expression after selection with acetamiprid $(\operatorname{logFC}=$ 2.45 in GR4-AS compared to GR4-AP). Over-expression of members of CYP4 family has been found associated with a number of insecticide resistant insect strains (reviewed in table 7 of [30], but also [77]) including neonicotinoids. For example, members of CYP4 (CYP4C1 and CYP4v2) family have been found over-expressed in MEAM1 B. tabaci resistant to thiamethoxam [29, 32], or induced after treatment with imidacloprid (CYP4G36) in Aedes aegypti [78], or in Diaphorina citri (CYP4C67, CYP4DA1, CYP4C68, CYP4DB1, and CYP4G70) [79].

Unigene comp50040_c0 had a significant Blast hit with CYP6CM1vQ of B. tabaci (100\% similarity) and was differentially unregulated in the three resistant strains with similar, very high expression levels, compared to the susceptible strain $(\log \mathrm{FC}=5.55-5.84)$.

CYP6 family P450s are widely known to be involved in metabolic resistance to pyrethroids and organophosphates in a number of insect species [80-83] including neonicotinoids $[74,75,84]$. CYP6CM1 was the first P450 isolated from an agricultural pest to be directly involved in neonicotinoid metabolism by recombinant expression. More specifically, it has been found able to metabolize imidacloprid, clothianidin and thiacloprid but not acetamiprid [10, 69]. It has been also found to metabolize the chemically unrelated insecticides pymetrozine [70] and pyriproxifen [85]. The cross resistance of acetamiprid-selected strain towards imidaclorpid (Table 1) could explain the high expression levels of CYP6CM1 in GR4-AS. Additionally, the comparable expression levels with the relaxed parental strain could mean that this P450 was initially selected in the field (due to selection by other neonicotinoids) and maintained the same expression levels after six generations in absence of any selection.

For components 57969_c113 and 57969_c124 the bidirectional best hits (1:1) were respectively CYP6CX5 and CYP6CX3 of B. tabaci. These two unigenes showed comparable over-expression levels in the three resistant strains $\quad(\log \mathrm{FC}=1.23-2.06$ for comp57969_c124 and $\log \mathrm{FC}=1.61-2.45$ for comp57969_c113). Although there is no significant difference, their expression levels slightly increase with acetamiprid selection in GR4_AS compared to GR4_AP. In the phylogenetic analysis, they were grouped together with the third member of clade CYP3 (CYP6CM1) that has also been found highly overexpressed in this study. All three (CYP6CX5, CYP6CX3 and CYP6CM1) displayed the higher similarity with the respective published sequences of $B$. tabaci as well as with CYP6CX1 and CYP6CX4. CYP6CX1 has been associated with $B$. tabaci (biotype B) resistance to fenvalerate, chlopyrifos, and avermectin in the field [86]. CYP6CX1 exhibited significantly higher mRNA expression in a laboratory-selected thiamethoxam resistant B. tabaci MEAM1 (B-biotype) strain [71].

All of the eight above mentioned P450s may be potentially associated with insecticide resistance and this could constitute a discrepancy compared to other insects, ie D. melanogaster where only a single P450, cyp6g1, is associated with neonicotinoid resistance [75]. However B. tabaci, is a heavily treated agricultural pest and different insecticides, of the same or different chemical groups, may select several P450s conferring unpredictable cross resistance. For example, CYP6CM1 the only functionally characterized P450 in B. tabaci, confers multiple resistant phenotypes and metabolizes some but not all neonicotinoids, as well as other insecticides from different chemical groups. Our differential expression study provides a good base to select additional candidate genes for further functional characterization and validate their role in resistant phenotypes.

\section{P450 expression levels in laboratory and field B. tabaci displaying different resistance phenotype using $q P C R$}

To further support P450s role as candidate genes we studied the association between resistant phenotype and expression levels. Focusing on P450 enzymes, we were able to set up a quantitative PCR for the seven of the eight higher up-regulated P450s (see previous paragraph) with $\log \mathrm{FC}>2$ (comp50040_c0, comp61334_c0, comp33028_c0, comp57969_c113, comp57969_c124, comp43065_c0 and comp43434_c1). Using this approach, we investigated their expression levels in several selected strains (some of them used in the RNA-seq experiment), as well as in additional whiteflies sampled in the field. The levels of gene expression (folds regulation) in resistant strains compared to the susceptible S_GR6 found in qPCRs were highly correlated with those observed in RNAseq experiment $\left(R^{2}=0.85 ; P<\right.$ 0.0001) (Fig. 5). This concordance between the transcription profiles obtained by the two techniques offered confidence to use qPCR and interpret the levels of expression of P450s in relation to neonicotinoid selection and resistance phenotype.

We first examined the levels of expression in comparisons between the strains maintained under neoinicotinoid selections and their respective relaxed strains (GR4_AS versus GR4_AP, GR9_IS versus GR9_IP and GR0_IS versus GR0-IP) (Fig. 5). In general, the P450 expression levels were higher in the strain GR4_AS, maintained under acetamiprid treatment, compared to its respective relaxed GR4_AP strain pointing out the involvement of these P450s in acetamiprid resistance. This has not been always the case for the two imidacloprid selected strains. More precisely, in these pair wise comparisons the laboratory acetamiprid treatment differentiated the expression levels for comp57969_c124, comp61334_c0 and comp43065_c0 but left unchanged 


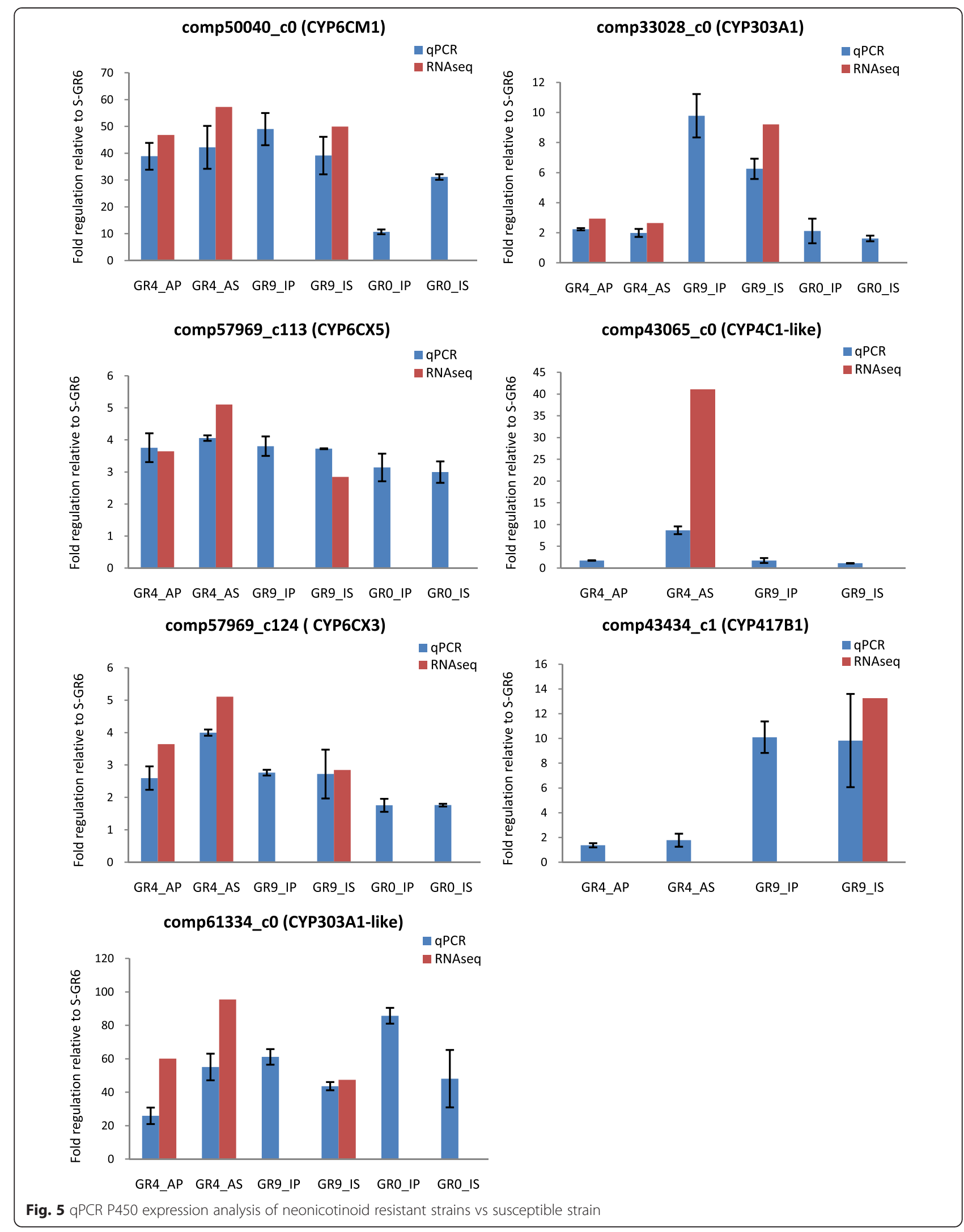


the levels of CYP6CM1, comp57969_c0, comp 33028 and comp43434 (Fig. 5). On the other hand, the pair wise comparisons disclosed that the imidacloprid treatment left the levels of expression unaffected for most of the unigenes except for CYP6CM1 that was higher in the selected counterpart in one of the two "imidacloprid" strain pairs (GR0_IS versus GR0_IP). This result could be explained by differences in phenotypic profiles toward the two neonicotinoids. Indeed, it is worth noting that although tolerance to both neonicotinoids was constantly higher in the strains maintained under treatment (GR0_AS, GR4_IS and GR9_IS), the confidence intervals (95\% CI) of Resistance Ratio (RR) in the imidacloprid selected strains (_IS) overlap with the CI of RR of their respective relaxed strains (Table 1 ) to both imidacloprid and acatamiprid. On the other hand, tolerance to acetamiprid and imidacloprid has been significantly higher in the acetamiprid selected strain (absence of CI overlap) compared to the parental one which has been maintained under the same conditions without acetamiprid (and any) treatment.

We subsequently scaled up and examined with qPCR the expression levels of these seven P450s, in five additional whitefly populations directly derived from the field. All populations belonged to MED B. tabaci and were collected from eggplants grown in greenhouses in Crete, setting the frequent application of neonicotinoids, pyrethroids, spiromesifen and pymetrozine as the common point in their treatment history. Compared to the laboratory susceptible strain S_GR6, their RR ranged from 6 to 65-fold for imidacloprid and 2 to 26.5-fold for acetamiprid (Additional file 14: Table S8). For three unigenes (com33028_c0, comp43065_c0 and comp43434_c1), relative over-expression of the P450s in the field populations, measured by qPCRs, reached a 2 -fold difference compared to S-GR6 while in unigenes comp57969_c113 and comp57969_c124 they increased 5.5-fold. Finally, in comp50040_c0 and comp61334_c0 the higher overexpression was detected with FC starting from 5 and reaching as high as 190-fold compared to the susceptible strain S_GR6 (Additional file 14: Table S8).

We then looked for the presence of correlation between resistant phenotypes and expression levels by plotting $\mathrm{RR}$ of all field derived and laboratory maintained strains against $\mathrm{qPCR}$ relative fold regulation. A positive correlation was observed between expression levels of comp61334_c0, CYP6CM1 and comp43065_c0 and $\mathrm{RR}$ to imidacloprid and between the expression of components 57969_c124 and 57969_c113 and RR to acetamiprid. However, a significant positive correlation has been found only between the expression of comp61334_c0 and RR to imidacloprid, and comp57969_c124 and RR to acetamiprid $\left(R^{2}=0.5, P=0.02 ; R^{2}=0.7, P<0.001\right.$, respectively) (Fig. 6).
In field MED (Q biotype) B. tabaci populations from China, levels of resistance to imidacloprid were correlated with expression levels of two P450s: CYP6CM1 and CYP4C64 [72]. In our RNA-seq data, comp47612_c0 with the best Blast hit to the CYP4C64 from B. tabaci appeared among the differentially over-expressed P450s in both acetamiprid selected and parental strain (GR4_AP and GR4_AS), with $\operatorname{logFC}$ close to 1 . For this reason, its expression levels were not examined in more populations by qPCR and we were are not able to assess the presence of any possible correlation with resistance levels in the Greek populations. Components 61334_c0 and 57969_c124 are the number one candidates for further studies, i.e. functional analysis, given their stronger positive correlation with the neonicotinoid resistant phenotype. However, the absence of significant correlation between RR and relative fold regulation cannot exclude the possibility of another P450 being involved in imidacloprid or acetamiprid resistance. Rather, it indicates that this $\mathrm{P} 450$ is probably not the major, in terms of frequency, resistance mechanism for the given populations. This is illustrated by the case of CYP6CM1 whose implication in neonicotinoid resistance has been functionally demonstrated; although in the present study, no significant correlation was found. It is worth noting the total absence of a correlation between CYP6CM1 and RR to acetamiprid is in agreement with the fact that acetamiptid is not metabolized by this P450. The unpredictable diverse range of chemistries detoxified by CYP6CM1, which includes some - but not all - neonicotinoids [10,69] as well as the unrelated chemistries of pymetrozine [70] and pyriproxyfen [85], exemplify the complex relationship between resistance and expression of a single P450. Our study clearly demonstrates that several P450s can simultaneously operate in a given resistant population and that expression profile can change between populations. This suggests that different $\mathrm{P} 450$ genes could be involved in different cases of neonicotinoid resistance.

\section{Conclusion}

Our work has generated new toxicological data and genomic resources which will significantly enrich the available dataset and substantially facilitate the molecular studies in MED B. tabaci. Twelve libraries from three neonicotinoid resistant $B$. tabaci strains from Greece were sequenced by Illumina platform, and generated about $73 \mathrm{Gbp}$. A reference transcriptome was built by de novo assembly and functionally annotated. The large number of sequences, obtained by the investigation of expression profile of different neonicotinoid resistant strains, provided useful insights into the underlying molecular mechanisms associated with resistance. We further focused on the differential expression analysis and phylogenetic classification of nAChR subunits and 


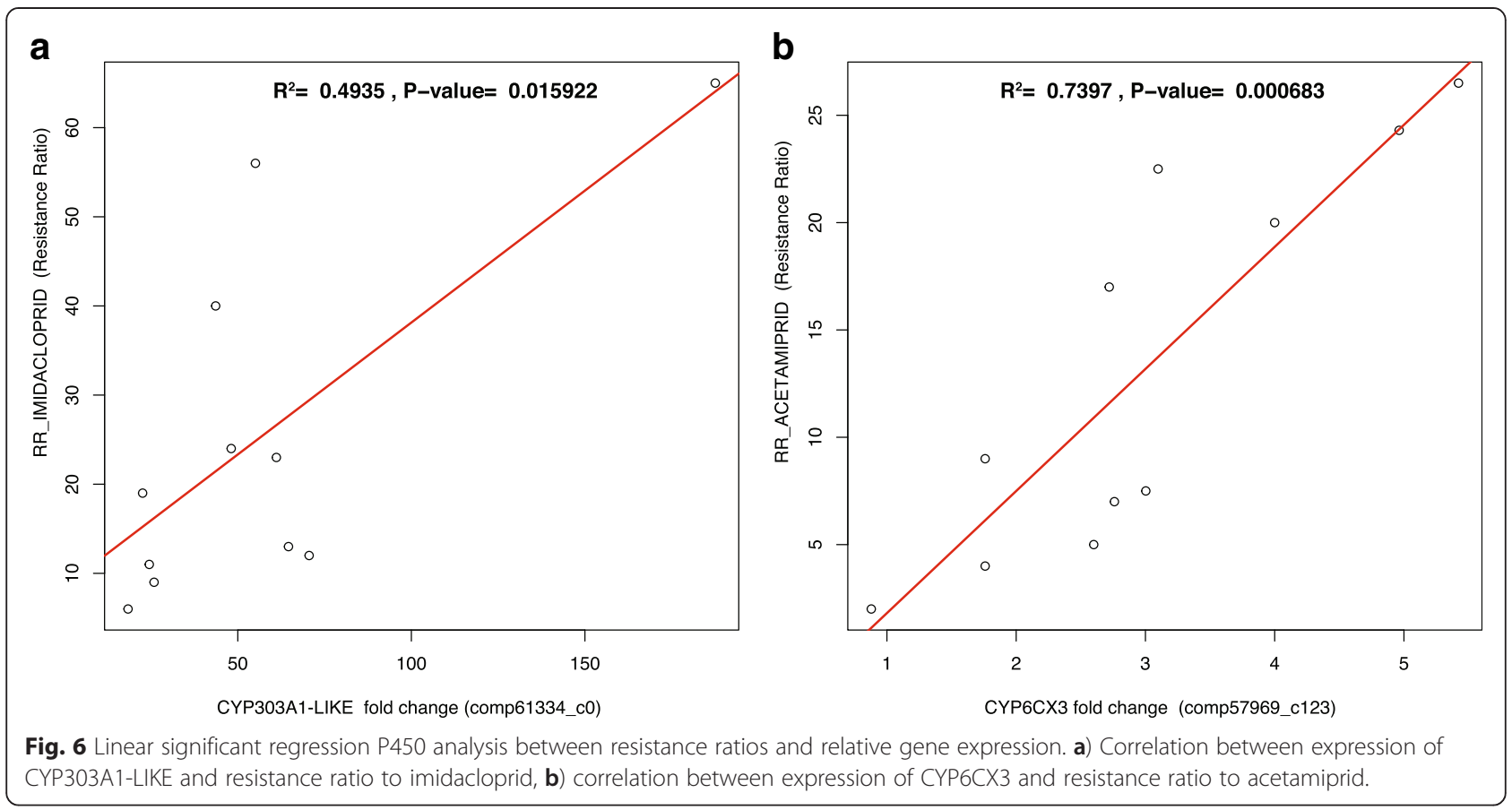

cytochrome P450s, the main genes related to neonicotinoid resistance, enabling useful genetic information of resistance mechanisms to be gained. No nAChR polymorphism or differential expression of the different nAChR subunits were observed among the studied strains that could be related to the resistant phenotypes. Differential expression analyses disclosed a number of significantly up regulated detoxification genes (mainly cytochrome P450s), some of which specifically overexpressed in each selected strain, providing a list of putative candidate resistance genes. CYP6CM1 already known as a major mechanism of imidacloprid resistance in B. tabaci from Crete was also deferentially expressed in the resistant strains. Using quantitative qPCRs, a larger screening of field MED B. tabaci from Crete with known neonicotinoid phenotype was performed to associate expression levels of P450s with resistance levels. Expression levels of five P450s including CYP6CM1 were found associated with neonicotinoid resistance, however, a significant correlation was found only in two of them, CYP303 and CYP6CX3, with imidacloprid and acetamiprid respectively. Further functional expression analysis of the genes and the encoded proteins are currently being investigated to confirm their role in resistance.

\section{Methods}

\section{Sample collection/biological material}

Three B. tabaci populations (GR0, GR4 and GR9), collected in 2012 in Crete on eggplant greenhouses were maintained in laboratory conditions under short term selections and were chosen based on their $\mathrm{LC}_{50} \mathrm{~S}$ (concentrations that cause $50 \%$ mortality) to neonicotinoids and their insecticide application history. Insecticide selection experiments were carried out in parallel for the three strains during six generations with the neonicotinoids acetamiprid (GR4_AS) and imidacloprid (GR9-IS and GR0-IS). During the experiment, the initial parental strains (GR4-AP, GR9-IP and GR0-IP) were also maintained without insecticide selection. Under these relaxed conditions, it is expected that the Resistance Ratio (RR) in the parental strains would be lower than the respective selected ones, given that a fitness cost is often associated to the insecticide resistance.

Five additional B. tabaci populations collected in Crete in 2013 from eggplants grown in greenhouses, were also used in our study without any prior rearing in the laboratory (BT1, BT2, BT3, BT4 and BT5).

All populations were identified as the Mediterranean species MED (Q1 mitotype) by sequencing part of their COI mtDNA gene and applying a diagnostic PCR to 50 females of each strain [87].

In all experiments, the laboratory MED strain S-GR6 susceptible to neonicotinoids was used as reference strain. It was collected in 2006 in Crete and has been since maintained in the lab without any insecticide treatment.

\section{Bioassays and selections}

The selected strains were established by $>500$ field collected individuals and were reared on cotton plants in standard insectary conditions $\left(23-26{ }^{\circ} \mathrm{C}, 14: 10 \mathrm{~h}\right.$ Light:Dark, 70 \% relative humidity). Selections were performed by exposing adult females and males for $72 \mathrm{~h}$ to a lethal 
concentration of commercial formulations of imidacloprid or acetamiprid. Survivors were transferred onto untreated cotton plants and allowed to lay eggs to start a new generation. The concentration of insecticide was adjusted at each generation (200 to $1200 \mu \mathrm{g} / \mathrm{L}$ imidacloprid, 100 to $1200 \mu \mathrm{g} / \mathrm{L}$ acetamiprid) in order to reach 60-80\% mortality after $72 \mathrm{~h}$ exposure. Surviving adults were transferred onto untreated cotton plants to obtain the next generation. In order to limit bottleneck effects, each generation was set off with $>300$ individuals.

Full dose bioassays on cotton leaf discs were performed with female whiteflies as previously described [88, 89]. The insecticides used were: the neonicotinoids imidacloprid $200 \mathrm{~g}$ litre $^{-1} \mathrm{SL}$ (Confidor, Bayer AG, Leverkusen Germany), acetamiprid 20 SG (Profil, Nippon CO LYD, Japan) and thiacloprid $240 \mathrm{~g}$ litre $^{-1}$ SC (CaLypso, Bayer AG, Leverkusen Germany). Final mortality was assessed after $72 \mathrm{~h}$. Lethal concentrations corresponding to $50 \%$ mortality $\left(\mathrm{LC}_{50}\right)$ and their $95 \%$ confident intervals $\left(\mathrm{CI}_{95 \%}\right)$ were then calculated with a probit approach for each strain using PoloPC (LeOra Software, Berkeley, CA). Resistance ratios $\left(\mathrm{RR}_{50}\right.$ based on $\mathrm{LC}_{50}$ values) were calculated by comparison to the susceptible strain.

Bioassays after exposure to piperonyl butoxide (PB, Sigma, UK), an inhibitor of the P450 dependent monooxygenases, were used to determine P450 metabolism contribution to resistant phenotypes. Piperonyl butoxide was applied at $300 \mathrm{ppm}$ via a tarsal contact method $4 \mathrm{~h}$ prior to the insecticide application as previously described [69].

\section{RNA isolation, library construction and sequencing}

In total, four different strains -all of MED species originated from Crete- were used in the RNA-Seq experiments: 1) the neonicotinoid susceptible strain S_GR6 was used as a reference strain 2) the resistant strain R_GR9_IS maintained under Imidacloprid Selection 3) the resistant strain R_GR4_AS maintained under Acetamiprid Selection, and 4) its corresponding Parental strain R_GR4_AP issued from the same field collected population (R_GR_4) which has been maintained in the laboratory without any selection pressure. Strains R_GR9_IS and R_GR4_AS were used as they exhibited the higher resistance levels against neonicotinoids.

Three biological replicates were included per strain in order to statistically validate the pair wise comparisons of transcript expression between the resistant and susceptible strains. For each biological replicate, total RNA was prepared by grinding approximately 300 frozen adults 1-2 days old, including males and females, using the Qiagen RNeasy mini kit according to the manufacturer's protocol. Additionally, column digestion with DNAseI (Qiagen) was applied for the removal of genomic DNA contaminations. RNA concentration as well as the $260 / 280$ and the $260 / 230$ ratios were estimated using a Nanodrop spectrophotometer after which RNA samples were subjected to quality check with an Agilent Biolanalyzer, taking into account that in insects (and other taxa) the electrophoresis profile of rRNA significantly differs from the standard benchmark [90].

RNA samples were sent to Macrogen (Korea) for mRNA paired-end library construction with the Illunina TruSeqTM RNA Sample Preparation Kits v2 following the manufacturer protocol (Poly-A mRNA isolation with oligo-dT beads, mRNA fragmentation then followed by transcription into first-strand cDNA using reverse transcriptase and random hexamer primers) and sequencing on Illumina HiSeq 2000 platform. Barcoded libraries were prepared in a way that six samples could be run per lane. Each library was sequenced with the pairedend method for a read length of 100 base pairs.

\section{Read pre-processing}

Read quality was assessed with FastQC [91] and PrinSeq [92]. For each sample, the raw reads were cleaned by removing adaptor sequences in two steps. First, using Scythe [93] to identify 3'-end adapters which often include poor quality bases. Then, SeqPrep [94] was used for both $5^{\prime}$ and $3^{\prime}$ adaptor removal. Low quality nucleotide reads trimming (Phred quality threshold of 25 and minimum reads length of $40 \mathrm{nt}$ ) was performed with Sickle, a sliding-window, adaptive, quality-based trimming tool for FastQ files (available at [95]). Finally, low complexity sequences (threshold entropy value of 60) and poly A/T 5 ' tail (minimum of $5 \mathrm{~A} / \mathrm{T}$ ) trimming was performed using PrinSeq. To estimate ribosomal and mitochondrial RNA and remove the contaminant rRNAs, NCBI rRNA from Aleyrodidae, the SILVA rRNA database version 111 [96] and NCBI Bemisia tabaci mitochondrial genome were used. The reads from the fastq file were firstly collapsed (fastx_collapser from the fastx toolkit [97] to remove exact duplicate reads thus reducing the sequence number and a fasta file was produced. Then, ribokliper [98] (BLAT as aligner) was used to identify contaminant RNAs. At the end, the reads of each pair of all the libraries were re-combined and the remaining unpaired reads (singletons) were merged in a separate file using custom Perl scripts.

\section{Assembly}

A preliminary assembly study was conducted in a subset of the dataset with SOAPdenovo-Trans [99] (kmers (1777 , steps of 2) optimal kmer 37, N50 $=500$, average contig length $=293$ and 214,371 contigs), Oases [100] (kmers (17-77, steps of 2) optimal kmer 35 , N50 = 150, average contig length 136 and 668,034 contigs) and Trinity [101] (fixed kmers 25, N50 = 1961, average contig length 1032 and 107,274 contigs). 
Based on the results of the preliminary assemblies, we selected to conduct our final de novo assembly using Trinity package. To increase transcriptome coverage and to facilitate downstream analyses (differential gene expression), de novo assembly was performed on a pool of the pre-processed reads (singleton and re-paired) from all the 12 samples. A single reference transcriptome was generated using Trinity package (release: 2013-02-25 with default parameters, kmers-25 and the minimum contig size fixed to $200 \mathrm{bp}$ ).

To assess the assembled transcripts quality, we followed four procedures: a) we pooled all the reads and mapped them to the assembly using Bowtie2 [102] and Samtools [103] to estimate the percentage of mapped reads, b) the quality of the assembled data was assessed by examining the similarity between the Trinity assembly and nr NCBI protein database using blastx search, c) the completeness of the produced contigs was estimated using a dataset of 129 complete mRNA of B. tabaci obtained from NCBI with blastn search, and d) with CEGMA (Core Eukaryotic Genes Mapping Approach) [104] we determined how many of 248 highly conserved eukaryotic genes were present in the $B$. tabaci transcriptome. In addition, based on BLAST NCBI taxonomy database we identified and removed 3, 467 host plant and fungus contaminant contigs (representing 2552 unigenes), as well as their respective mapped reads, from further analysis. No filtering against low-abundance contigs was performed in order to maximize the detection of low expressed transcripts. All the BLAST (http://www.ncbi.nlm.nih.gov/pubmed/ 2231712) searches were performed using NOBLAST program [105] (http://sourceforge.net/projects/noblast/).

\section{Homology searches and sequencing annotation}

For sequence annotation of the assembled transcripts, first, a blastx similarity search against the NCBI protein database $n r$ (e-value threshold $10^{-6}$; keeping the top 20 hits) was performed. Sequences that did not receive any hit were then searched via blastn against NCBI nucleotide database $n t$ (e-value threshold $10^{-10}$; keeping the top 20 hits) using the parallel version of NOBLAST. For gene ontology (GO) mapping, Blast2GO [106] was used locally to recover all the GO terms associated to the hits obtained by the blast search. After the mapping step, results were subjected to GO annotation, a process of selecting GO terms from the GO pool and assigning them to the query sequences.

The sequences were further annotated using InterPro [107]. A local InterProScan (version 5, InterPro release 42.0) [108] was run in parallel (splitting the query set) on the longest open reading frame (ORF) of the contigs using a custom pair script based on the EMBOSS program "getorf" [109]. GO terms corresponding to these InterPro domains, were merged with the already existent GO terms derived from the blastx against $n r$.
GO terms were modulated using the annotation augmentation tool ANNEX [110] followed by GOSlim [111]. Enzyme classification (EC) codes were obtained through the direct mapping of GO terms to the corresponding enzyme codes. Sequences having EC numbers were further characterized by Kyoto Encyclopedia of Genes and Genomes (KEGG) [112] metabolic pathway annotations using custom perl/R scripts.

\section{P450s and nAChR similarity searches and phylogenetic inferences}

For genes of particular interest expected to be involved in neonicotinoid resistance such as P450s and nAChR, similarity search and phylogenetic reconstructions were performed using known orthologs from other insect species.

B. tabaci contigs (>200 bp) encoding P450s and nAChRs were identified using BLAST, Blast2Go and interpro as described above. In addition, P450s and nAChRs were further identified using tblastn search $(e$-value cutoff $<10^{-10}$ ) in protein sequences of other insect species from $[33,38,113,114]$ or downloaded from GenBank/ ENSEMBL used as query against the $B$. tabaci assembly transcriptome. Contigs were translated and searched by Blastp against themselves. Results showing more than $99 \%$ similarity were considered as allelic variants. Specifically for nAChR subunits, which displayed high expression variation (see Results and Discussion for details), the nucleotide sequence length of contigs included in phylogenetic analysis ranged from $346 \mathrm{bp}$ ( $\alpha 6$ subunit) to 5,473 bp ( $\alpha 4$ subunit), with an average of $1,789 \mathrm{bp}$.

MAFFT-7.050 [115] was used to perform multiple sequence alignment of $B$. tabaci MED P450 protein sequences with a representative dataset of their counterparts in other species (Additional file 15: Figure S7).

Only protein sequences showing no misalignment were used in the final dataset for phylogenetic analysis.

Model selection was performed with ProtTest-3 [116] and the optimum model for phylogenetic analysis was selected according to Akaike information criterion (P450s: $L G+G+F$, nAChR: WAG + G + F). A maximum likelihood analysis was performed using RaxML v8 [117] and a bootstrap analysis with 1000 pseudoreplicates was performed to evaluate the branch strength of each tree. The resulting tree was midpoint rooted and edited with FigTree-1.4.1 software [118].

\section{Detection of polymorphism in the $\mathrm{nAChR}$}

Detection of single nucleotide polymorphisms (SNPs) was performed for the nAChR genes. Among all detected polymorphism variations, only SNP substitutions were considered for differential polymorphism analyses. Alignment files generated by Bowtie2 were used for SNP detection using SAMtools and Varscan [119] with the following parameters: map quality $>10$, PHRED quality 
score $>25$, coverage $>10$ reads, $P$ value threshold for calling variant $=0.01$ and minimum supporting reads at a position to call variant $=4$.

SNP allele frequencies were then computed between each insecticide resistant strain and the susceptible strain. Allele frequencies were considered as differential between an insecticide-selected strain and the susceptible strain (differential SNPs) if the total read coverage at SNP position between both strains was $\geq 50$ and difference in allele frequency between strains > $50 \%$ [22].

SNPs were categorized to synonymous, nonsynonymous and those that belong to the transcripts UTRs using a custom perl script. Non-synonymous SNPs were manually checked.

\section{Differential gene expression analysis}

Differential expression (DE) analysis was performed at the "gene" (unigene) level by pair wise comparisons between strains. For the quantification, the paired reads of each sample were mapped to the transcriptome assembly with Bowtie2 and abundance was estimated with RSEM v. 1.2.4, as implemented in the trinity script run_RSEM_align_n_estimate.pl. The estimated expected counts for each sample (the sum of counts in each row $>10$ ) were extracted and used for the analysis of differential expression conducted in EdgeR-3.8.0 bioconductor package [120] using EdgeR-robust method to dampen the effect of outlier genes [121] with $\log \mathrm{FC}>1$ and FDR $<0.05$.

\section{Real time polymerase chain reaction}

The expression levels of seven P450 genes found to be differentially expressed by the RNA-seq approach were validated using quantitative reverse transcription PCR (qRT-PCR). Primers were designed using the Oligo7 Primer Analysis Software (Molecular Biology Insights, Cascade, CO, USA), based on cDNA sequences retrieved from RNA-seq and are listed in Additional file 16: Table S9. The qRT-PCRs were performed using as template the same RNA used for the library construction. An aliquot of $2.8 \mu \mathrm{g}$ total RNA from each of the three biological replicates, for each strain, served as a template for cDNA synthesis with Superscript III (Invitrogen, Carlsbad, CA, USA) using oligo- $\mathrm{dT}_{20}$, according to the manufacturer's instructions. The resulting cDNAs were diluted 20 times in ultra-high quality water for $\mathrm{qRT}-\mathrm{PCR}$ reactions using a Mini Opticon System (Biorad, Hercules, CA, USA). PCR reactions of $25 \mu \mathrm{l}$ contained Fast Start SYBR Green Master Mix (Kapa-Biosystems), $0.12 \mu \mathrm{M}$ of each primer (Additional file 16: Table S9) and $5 \mu \mathrm{l}$ of diluted cDNA. Melt curve analysis was performed to test the specificity of amplicons. A serial dilution of cDNA was used to generate standard curves for each gene in order to assess PCR efficiency and quantitative differences among samples. The fold-change of each target gene, normalized to the $60 \mathrm{~S}$ ribosomal protein L29 (RPL29), and relative to the susceptible reference strain S-GR6, was calculated according to the $2^{-\Delta \Delta C T}$ method incorporating PCR efficiency [122].

All the computations were performed at the HCMRCrete, high-performance computing bioinformatics platform. All the custom Perl, R and bash scripts are available from the authors upon request.

\section{Additional files}

\begin{abstract}
Additional file 1: Table S1. Statistics summary of library reads. (DOCX $14 \mathrm{~kb}$ )

Additional file 2: Table S2. Assembly statistics summary. (DOCX $12 \mathrm{~kb}$ ) Additional file 3: Figure S1. GO analysis by ontology category (level 2). A. Molecular function (MF); B. Biological process (BP); C. Cellular component (CC). (A)The majority of the MF are involved in "catalytic activity" (41.25\%) and "binding" (39.93\%), followed by "transporter activity" (6.9\%). (B)The largest subcategories found in the BP group were "metabolic process" (17.7 \%) and "cellular process" (17.7\%). The largest subcategory found in CC group was "cell" which comprised $43.4 \%$ of the contigs followed by "organelle" (24.12\%). (PDF 50 kb)
\end{abstract}

Additional file 4: Table S3. Summary of KEGG pathway mapping of B. tabaci contigs. (XLSX $13 \mathrm{~kb}$ )

Additional file 5: Figure S2. Amino acid alignment of $n A C h R$ subunits from $B$. tabaci transcriptome with available protein sequences from 7 insect species. List of species in the alignment: D. Melanogaster (Dmel), A. mellifera (Amel), T. castaneum (Tcas), A. gambiae (Agamb), B. mori (Bmori), A. pisum (Apisum), B. tabaci (Btab). (Examination of this file requires the BioEdit program (http://www.mbio.ncsu.edu/bioedit/bioedit.html)). (ALI 135 kb)

Additional file 6: Figure S3. Amino acid alignment of B. tabaci nAChR subunits. Protein sequence alignment of $B$. tabaci nAChR subunits with D_a1 subunit of $D$. melanogaster. The numbers on the right indicate the amino acid position. The N-terminal signal peptides, the N-terminal region encompassing the six conserved domains loops (A-F) and the transmebrane domains were predicted by sequence similarity and using the SignalP4.1 [123] and TMHMM softwares [124]. Predicted N-terminal signal peptide leader sequences are shaded in yellow while the six ligand-binding loops $(D, A, E, B, F, C)$ and the four transmembrane domains (TM1-4) are shaded in grey and green respectively. Alignment of predicted protein sequences (aa sequence average length for all subunits except $a 6=457$; for $a 6=115$ ) shows that all subunits possess two Cys separated by 13 residues, typical of LGIC subunits, as well as six conserved Loops (Loops A-F) located at the N-terminal domain. All subunits carry a predicted signal peptide at the $\mathrm{N}$-terminus and four transmembrane motifs (TM1-TM4) at the C-terminal domain, which are involved in forming an ion channel. Loops between TM3 and TM4 were highly variable. The YxCC and GEK motifs are shaded in black. Amino acid mutations involved in imidacloprid resistance (R81T ( $M$. persicae numbering), Y151S (N. lugens numbering)) are marked by inverted red triangle while non-synonymous SPNs found in B. tabaci strains are shaded in blue and marked with asterisk. (PDF $39 \mathrm{~kb}$ )

Additional file 7: Figure S4. PCA plot. Overall similarity between samples using mapped reads on all contigs of the reference transcriptome (DESeq). The global gene expression pattern shows a clear separation between the susceptible and the three resistant strains. The higher correlation was observed among biological replicates of the same strain followed by the correlation between samples of the GR4_AP and the GR4_AS strains. The higher variance was found within strain R_GR4_AS: one biological replicate is closer to the parental strain R_GR4_AP while the two others are separated indicating signals of selection under insecticide pressure. (PDF $17 \mathrm{~kb}$ )

Additional file 8: Table S4. Differentially expressed unigenes (LogFC > 1, FDR < 0.05), with LogCPM and TMM normalized FPKM for each library with best blast hit. (XLSX $1123 \mathrm{~kb})$ 
Additional file 9: Figure S5. Heatmap spearman correlation matrix of significant DE (LogFC > 1 FDR < 0.05) unigenes based on their TMM-FPKM and $\mathrm{GO}$ enrichment analysis. The relative expression levels of each gene (row) in the susceptible and resistant samples (column) are shown. Upand down-regulated genes are represented respectively by red and yellow. Clustering analysis was performed using Euclidean distance and dward algorithm with optimal leaf order (unigenes) or Spearman's rank correlation (12 samples) using mean-centering transformation. The cluster was cut in three different parts using "cutree" and the unigenes of each sub cluster were recovered for GO enrichment analysis with Blast2GO for GO enrichment analysis using all DE as reference. Clustering of the samples grouped the neonicotinoid resistant samples together. Based on the transcription level of the 3,745 DE unigenes, three sub-clusters showing marked differences in normalized FPKM were identified. Assigning known transcripts to molecular functions revealed differences in the $\mathrm{GO}$ enrichment in the three clusters, displayed in the figure. The sub-clusters A1 and A2 group unigenes with lower expression profile in the susceptible strain and differ in expression profiles between the resistant strains. The sub-cluster A1 includes transcripts highly over-expressed in GR4-AS and GR4-AP and the more frequent GO terms included transferase activity (24\%), ATP binding (23\%) and heme binding (20\%). The sub-cluster A2 displayed the more visible and more abundant differences between the GR9-IS and the other resistant strains and revealed an enrichment in terms related to cytochrome P450s such as heme binding, iron ion binding and oxidoreductase activity (60\% of all overrepresented GO terms). Terms related to hydrolase activity were also enriched in sub-cluster A2. The cluster $B$ represents unigenes mostly up regulated in the susceptible strain. The cluster B is the most diversified with 16 GO terms enriched, the majority of which were related with signal transduction. (PDF $261 \mathrm{~kb}$ )

Additional file 10: Table S5. GO enrichment analysis of all strains vs full transcriptome. The file consists separate sheets of up and down regulated $\mathrm{GO}$ enrichment terms of each resistant strain (GR4-AP, GR4-AS, GR9-IS) vs susceptible strain S-GR6 and of GR4-AS vs GR4-AP against full transcriptome. (XLS $621 \mathrm{~kb}$ )

Additional file 11: Table S6. List of DE detoxification unigenes overexpressed in the three resistant strains. (XLSX 27 kb)

Additional file 12: Table S7. List of contigs encoding P450 genes in the B. tabaci transcriptome. (XLSX $48 \mathrm{~kb}$ )

Additional file 13: Figure S6. Phylogenetic analysis of B. tabaci P450s. Maximum likelihood phylogenetic tree of B. tabaci Med (BtMed, indicated in red) and P450 protein sequences from 14 insect species. Sequences were aligned using mafft and a bootstrapped midpoint-rooted tree was constructed (1000 bootstraps). Bootstrap percentage is indicated for crucial branches. List of species in the figure: D. melanogaster (Dmel), L. striatellus (Lstri), B. dorsalis (Bdor), D. citri (Dcit), H. zea (Hzea), D. punctata (Dpun), A. gossypii (Agoss), A. gambiae (Agam), M. Domestica (Mdom), P. polyxenes (Ppol), A. pisum (Apisum), A. melifera (Amel), M. persicae (Mpersi), B. tabaci (Btab). For accession numbers see tree or Additional file 15: Figure S7. (PDF 1005 kb)

Additional file 14: Table S8. Quantitative PCR analysis table of 7 P450s expression levels in five field populations of Bemisia tabaci and their resistance ratios to imidacloprid and acetamiprid, compared to the susceptible strain S-GR6. (DOCX $12 \mathrm{~kb}$ )

Additional file 15: Figure S7. Amino acid sequences (fasta format) of P450s from B. tabaci transcriptome with available protein sequences from other insect species included in the phylogeny (Additional file 13: Figure S6). (FAS $223 \mathrm{~kb}$ )

Additional file 16: Table S9. List of primer used in real-time quantitative PCR. (DOCX $12 \mathrm{~kb})$

\section{Competing interests}

The authors declare that they have no competing interests.

\section{Authors' contributions}

AT, JL and JV conceived and designed the study. Al prepared the RNA samples for RNAseq and performed the qPCRs. JL, Al and AT analysed data. AT, JL, Al and JV drafted the manuscript. Al, DEK and ER conducted whitefly sampling, toxicological assays and artificial selections. CST contributed in differential expression analysis. All authors read and approved the final manuscript.

\section{Acknowledgements}

We thank Mariana Stavrakaki, Maria Grispou and Natasa Fytrou for their assistance in experiments and Tereza Manousaki for her helpful comments on the manuscript. The authors are grateful to Dr Cameron Brown (Cyprus University of Technology) for improving the use of English in the latest version of the manuscript. This research has been co-financed by the European Union (European Social Fund - ESF) and Greek national funds through the Operation Program "Education and Lifelong Learning" of the National Strategic Reference Framework (NSRF)—Research Funding Program: THALES - MIS377301. We also acknowledge partial support by the LifeWatchGreece project (GSRT, structural funds) and ERA-NET Grant Agreement ARIMNET SWIPE KBBE 219262.

\section{Author details}

${ }^{1}$ Hellenic Agricultural Organisation - "DEMETER", NAGREF - Institute of Olive Tree, Subtropical Crops and Viticulture, Heraklion, Greece. ${ }^{2}$ Institute of Marine Biology, Biotechnology and Aquaculture (IMBBC), Hellenic Centre for Marine Research (HCMR), Heraklion, Greece. ${ }^{3}$ Department of Environmental and Natural Resources, University of Patras, Agrinio, Greece. ${ }^{4}$ Department of Crop Science, Agricultural University of Athens, Athens, Greece. ${ }^{5}$ Institute of Molecular Biology and Biotechnology, Foundation of Research and Technology, Heraklion, Greece.

Received: 6 June 2015 Accepted: 29 October 2015 Published online: 14 November 2015

\section{References}

1. Lee W, Park J, Lee G-S, Lee S, Akimoto S-I. Taxonomic status of the Bemisia tabaci complex (Hemiptera: Aleyrodidae) and reassessment of the number of its constituent species. PLoS One. 2013;8(5):e63817.

2. De Barro PJ, Liu S-S, Boykin LM, Dinsdale AB. Bemisia tabaci: a statement of species status. Annu Rev Entomol. 2011;56(1):1-19.

3. Horowitz AR, Kontsedalov S, Khasdan V, Ishaaya I. Biotypes B and Q of Bemisia tabaci and their relevance to neonicotinoid and pyriproxyfen resistance. Arch Insect Biochem Physiol. 2005;58(4):216-25.

4. Roditakis E, Grispou M, Morou E, Kristoffersen JB, Roditakis N, Nauen R, et al. Current status of insecticide resistance in Q biotype Bemisia tabaci populations from Crete. Pest Manag Sci. 2009;65(3):313-22.

5. Vassiliou V, Emmanouilidou M, Perrakis A, Morou E, Vontas J, Tsagkarakou A, et al. Insecticide resistance in Bemisia tabaci from Cyprus. Insect Sci. 2011;18(1):30-9.

6. Dennehy TJ, Degain BA, Harpold VS, Zaborac M, Morin S, Fabrick JA, et al. Extraordinary resistance to insecticides reveals exotic $\mathrm{Q}$ biotype of Bemisia tabaci in the New World. J Econ Entomol. 2010;103(6):2174-86.

7. Wang Z, Yan H, Yang $Y$, Wu Y. Biotype and insecticide resistance status of the whitefly Bemisia tabaci from China. Pest Manag Sci. 2010;66(12):1360-6.

8. Elbert A, Haas M, Springer B, Thielert W, Nauen R. Applied aspects of neonicotinoid uses in crop protection. Pest Manag Sci. 2008;64(11):1099-105.

9. Liu Z, Williamson MS, Lansdell SJ, Denholm I, Han Z, Millar NS. A nicotinic acetylcholine receptor mutation conferring target-site resistance to imidacloprid in Nilaparvata lugens (brown planthopper). Proc Natl Acad Sci U S A. 2005;102(24):8420-5.

10. Karunker I, Morou E, Nikou D, Nauen R, Sertchook R, Stevenson BJ, et al. Structural model and functional characterization of the Bemisia tabaci CYP6CM1VQ, a cytochrome P450 associated with high levels of imidacloprid resistance. Insect Biochem Mol Biol. 2009;39(10):697-706.

11. Karunker I, Benting J, Lueke B, Ponge T, Nauen R, Roditakis E, et al. Overexpression of cytochrome P450 CYP6CM1 is associated with high resistance to imidacloprid in the B and Q biotypes of Bemisia tabaci (Hemiptera: Aleyrodidae). Insect Biochem Mol Biol. 2008;38(6):634-44.

12. Gauthier N, Clouet C, Perrakis A, Kapantaidaki D, Peterschmitt M, Tsagkarakou A. Genetic structure of Bemisia tabaci Med populations from home-range countries, inferred by nuclear and cytoplasmic markers: impact on the distribution of the insecticide resistance genes. Pest Manag Sci. 2014;70(10):1477-91. 
13. Ilias A. Investigation of insecticide resistance mechanisms and geographical distribution of responsible genes in the whitefly Bemisia tabaci and the spider mite Tetranychus urticae. PhD thesis. Department of Biology, University of Crete; 2014

14. Crawford JE, Guelbeogo WM, Sanou A, Traoré A, Vernick KD, Sagnon NF, et al. De Novo transcriptome sequencing in Anopheles funestus using illumina RNA-Seq technology. PLoS One. 2010;5(12):e14202.

15. Wang X-W, Luan J-B, Li J-M, Bao Y-Y, Zhang C-X, Liu S-S. De novo characterization of a whitefly transcriptome and analysis of its gene expression during development. BMC Genomics. 2010;11(1):400.

16. Xue J, Bao Y-Y, Li B-I, Cheng Y-B, Peng Z-Y, Liu H, et al. Transcriptome analysis of the brown planthopper Nilaparvata lugens. PLoS One. 2010;5(12):e14233.

17. Chen S, Yang P, Jiang F, Wei Y, Ma Z, Kang L. De Novo analysis of transcriptome dynamics in the Migratory Locust during the development of phase traits. PLoS One. 2010;5(12):e15633.

18. Price DP, Nagarajan V, Churbanov A, Houde P, Milligan B, Drake LL, et al The fat body transcriptomes of the yellow fever mosquito Aedes aegypti, pre- and post- blood meal. PLoS One. 2011;6(7):e22573.

19. Karatolos N, Pauchet Y, Wilkinson P, Chauhan R, Denholm I, Gorman K, et al. Pyrosequencing the transcriptome of the greenhouse whitefly, Trialeurodes vaporariorum reveals multiple transcripts encoding insecticide targets and detoxifying enzymes. BMC Genomics. 2011;12(1):56

20. Liu B, Jiang G, Zhang Y, Li J, Li X, Yue J, et al. Analysis of transcriptome differences between resistant and susceptible strains of the citrus red mite Panonychus citri (Acari: Tetranychidae). PLoS One. 2011;6(12):e28516.

21. Xie W, Yang X, Wang S-I, Wu Q-j, Yang N-n, Li R-m, et al. Gene expression profiling in the thiamethoxam resistant and susceptible B-biotype sweetpotato whitefly, Bemisia tabaci. J Insect Sci. 2012;12(46):1-14

22. David J-P, Faucon F, Chandor-Proust A, Poupardin R, Riaz M, Bonin A, et al. Comparative analysis of response to selection with three insecticides in the dengue mosquito Aedes aegypti using mRNA sequencing. BMC Genomics. 2014;15(1):174

23. Mamidala P, Wijeratne A, Wijeratne S, Kornacker K, Sudhamalla B, RiveraVega $L$, et al. RNA-Seq and molecular docking reveal multi-level pesticide resistance in the bed bug. BMC Genomics. 2012;13(1):6.

24. Xia J, Zhang C-R, Zhang S, Li F-F, Feng M-G, Wang X-W, et al. Analysis of whitefly transcriptional responses to Beauveria bassiana infection reveals new insights into insect-fungus interactions. PLoS One. 2013;8(7):e68185.

25. Su Y-L, Li J-M, Li M, Luan J-B, Ye X-D, Wang X-W, et al. Transcriptomic analysis of the salivary glands of an invasive whitefly. PLoS One. 2012;7(6):e39303.

26. Wang X-W, Luan J-B, Li J-M, Su Y-L, Xia J, Liu S-S. Transcriptome analysis and comparison reveal divergence between two invasive whitefly cryptic species. BMC Genomics. 2011;12(1):458.

27. Wang X-W, Zhao Q-Y, Luan J-B, Wang Y-J, Yan G-H, Liu S-S. Analysis of a native whitefly transcriptome and its sequence divergence with two invasive whitefly species. BMC Genomics. 2012;13(1):529.

28. Ye X-D, Su Y-L, Zhao Q-Y, Xia W-Q, Liu S-S, Wang X-W. Transcriptomic analyses reveal the adaptive features and biological differences of guts from two invasive whitefly species. BMC Genomics. 2014;15(1):370.

29. Yang N, Xie W, Jones CM, Bass C, Jiao X, Yang X, et al. Transcriptome profiling of the whitefly Bemisia tabaci reveals stage-specific gene expression signatures for thiamethoxam resistance. Insect Mol Biol. 2013;22(5):485-96

30. Feyereisen R. Insect CYP genes and P450 enzymes. In: Gilbert LI, editor. Insect molecular biology and biochemistry. San Diego: Academic; 2012. p. 236-316.

31. Hsu J-C, Chien T-Y, Hu C-C, Wu W-J, Chen M-JM, Feng H-T, et al. Discovery of genes related to insecticide resistance in Bactrocera dorsalis by functional genomic analysis of a De Novo assembled transcriptome. PLoS One. 2012;7(8):e40950.

32. Xie W, Meng Q-S, Wu Q-j, Wang S-I, Yang X, Yang N-n, et al. Pyrosequencing the Bemisia tabaci transcriptome reveals a highly diverse bacterial community and a robust system for insecticide resistance. PLoS One. 2012;7(4):e35181

33. Pavlidi N, Dermauw W, Rombauts S, Chrisargiris A, Van Leeuwen T, Vontas J. Analysis of the olive fruit fly Bactrocera oleae transcriptome and phylogenetic classification of the major detoxification gene families. PLoS One. 2013;8(6):e66533.
34. Jeschke P, Nauen R. Neonicotinoids - from zero to hero in insecticide chemistry. Pest Manag Sci. 2008;64(11):1084-98.

35. Jones A, Sattelle D. Diversity of insect nicotinic acetylcholine receptor subunits. In: Thany S, editor. Insect nicotinic acetylcholine receptors, vol. 683. New York: Springer; 2010. p. 25-43.

36. Shao Y-M, Dong K, Zhang C-X. The nicotinic acetylcholine receptor gene family of the silkworm, Bombyx mori. BMC Genomics. 2007;8(1):324.

37. Dupuis J, Louis T, Gauthier M, Raymond V. Insights from honeybee (Apis mellifera) and fly (Drosophila melanogaster) nicotinic acetylcholine receptors: from genes to behavioral functions. Neurosci Biobehav Rev. 2012;36(6):1553-64.

38. Dale RP, Jones AK, Tamborindeguy C, Davies TGE, Amey JS, Williamson S, et al. Identification of ion channel genes in the Acyrthosiphon pisum genome. Insect Mol Biol. 2010;19:141-53.

39. Rinkevich FD, Scott JG. Transcriptional diversity and allelic variation in nicotinic acetylcholine receptor subunits of the red flour beetle, Tribolium castaneum. Insect Mol Biol. 2009;18(2):233-42.

40. Grauso M, Reenan RA, Culetto E, Sattelle DB. Novel putative nicotinic acetylcholine receptor subunit genes, Da5, Da6 and Da7, in Drosophila melanogaster identify a new and highly conserved target of adenosine deaminase acting on RNA-mediated A-to-I Pre-mRNA editing. Genetics. 2002;160(4):1519-33.

41. Lansdell SJ, Millar NS. Cloning and heterologous expression of Da4, a Drosophila neuronal nicotinic acetylcholine receptor subunit: identification of an alternative exon influencing the efficiency of subunit assembly. Neuropharmacology. 2000;39(13):2604-14.

42. Jin Y, Tian N, Cao J, Liang J, Yang Z, LV J. RNA editing and alternative splicing of the insect nAChR subunit alpha6 transcript: evolutionary conservation, divergence and regulation. BMC Evol Biol. 2007;7(1):98.

43. Jones AK, Raymond-Delpech V, Thany SH, Gauthier M, Sattelle DB. The nicotinic acetylcholine receptor gene family of the honey bee, Apis mellifera. Genome Res. 2006;16(11):1422-30.

44. Yao X, Song F, Zhang Y, Shao Y, Li J, Liu Z. Nicotinic acetylcholine receptor $\beta 1$ subunit from the brown planthopper, Nilaparvata lugens: A-to-I RNA editing and its possible roles in neonicotinoid sensitivity. Insect Biochem Mol Biol. 2009;39(5-6):348-54.

45. Taillebois E, Beloula A, Quinchard S, Jaubert-Possamai S, Daguin A, Servent $D$, et al. Neonicotinoid binding, toxicity and expression of nicotinic acetylcholine receptor subunits in the aphid Acyrthosiphon pisum. PLoS One. 2014;9(5):e96669.

46. Yu X, Wang M, Kang M, Liu L, Guo X, Xu B. Molecular cloning and characterization of two nicotinic acetylcholine receptor $\beta$ subunit genes from Apis cerana cerana. Arch Insect Biochem Physiol. 2011;77(4):163-78.

47. Markussen MDK, Kristensen M. Low expression of nicotinic acetylcholine receptor subunit Mda2 in neonicotinoid-resistant strains of Musca domestica L. Pest Manag Sci. 2010;66(11):1257-62.

48. Bass C, Puinean A, Andrews M, Cutler P, Daniels M, Elias J, et al. Mutation of a nicotinic acetylcholine receptor beta subunit is associated with resistance to neonicotinoid insecticides in the aphid Myzus persicae. BMC Neurosci. 2011;12(1):51.

49. Grutter T, Changeux J-P. Nicotinic receptors in wonderland. Trends Biochem Sci. 2001;26(8):459-63.

50. Tricoire-Leignel $H$, Thany S. Identification of critical elements determining toxins and insecticide affinity, ligand binding domains and channel properties. In: Thany S, editor. Insect nicotinic acetylcholine receptors, vol. 683. New York: Springer; 2010. p. 45-52.

51. McCarthy DJ, Chen Y, Smyth GK. Differential expression analysis of multifactor RNA-Seq experiments with respect to biological variation. Nucleic Acids Res. 2012;40(10):4288-97.

52. Tsagkarakou A, Mouton L, Kristoffersen JB, Dokianakis E, Grispou M, Bourtzis K. Population genetic structure and secondary endosymbionts of Q Bemisia tabaci (Hemiptera: Aleyrodidae) from Greece. Bull Entomol Res. 2012;102(03):353-65.

53. Vontas JG, Small GJ, Hemingway J. Glutathione S-transferases as antioxidant defence agents confer pyrethroid resistance in Nilaparvata lugens. Biochem J. 2001;357(Pt 1):65-72.

54. David J-P, Strode C, Vontas J, Nikou D, Vaughan A, Pignatelli PM, et al. The Anopheles gambiae detoxification chip: a highly specific microarray to study metabolic-based insecticide resistance in malaria vectors. Proc Natl Acad Sci U S A. 2005;102(11):4080-4. 
55. Reyes M, Bouvier JC, Boivin T, Fuentes-Contreras E, Sauphanor B. Susceptibilidad a Insecticidas y Actividad Enzimática de Cydia pomonella L. (Lepidoptera: Tortricidae) Proveniente de Tres Huertos de la Región del Maule, Chile. Agricultura Técnica. 2004;64:229-37.

56. Reyes M, Franck P, Charmillot P-J, loriatti C, Olivares J, Pasqualini E, et al. Diversity of insecticide resistance mechanisms and spectrum in European populations of the codling moth, Cydia pomonella. Pest Manag Sci. 2007;63(9):890-902.

57. Hemingway J. The molecular basis of two contrasting metabolic mechanisms of insecticide resistance. Insect Biochem Mol Biol. 2000;30(11):1009-15.

58. Ono M, Swanson JJ, M. Field L, Devonshire AL, D. Siegfried B. Amplification and methylation of an esterase gene associated with insecticide-resistance in greenbugs, Schizaphis graminum (Rondani) (Homoptera: Aphididae). Insect Biochem Mol Biol. 1999;29(12):1065-73.

59. Small GJ, Hemingway J. Molecular characterization of the amplified carboxylesterase gene associated with organophosphorus insecticide resistance in the brown planthopper, Nilaparvata lugens. Insect Mol Biol. 2000;9(6):647-53.

60. Vontas J, Kioulos E, Pavlidi N, Morou E, della Torre A, Ranson H. Insecticide resistance in the major dengue vectors Aedes albopictus and Aedes aegypti. Pestic Biochem Physiol. 2012;104(2):126-31.

61. David J-P, Ismail HM, Chandor-Proust A, Paine MJI. Role of cytochrome P450s in insecticide resistance: impact on the control of mosquito-borne diseases and use of insecticides on Earth. Philosophical Transactions of the Royal Society B: Biological Sciences. 2013;368:1612.

62. Wheelock GD, Scott JG. The role of cytochrome P450 Ipr in deltamethrin metabolism by pyrethroid-resistant and susceptible strains of house flies. Pestic Biochem Physiol. 1992:43(1):67-77.

63. Joußen N, Heckel DG, Haas M, Schuphan I, Schmidt B. Metabolism of imidacloprid and DDT by P450 CYP6G1 expressed in cell cultures of Nicotiana tabacum suggests detoxification of these insecticides in Cyp6g1overexpressing strains of Drosophila melanogaster, leading to resistance. Pest Manag Sci. 2008;64(1):65-73.

64. Ding Z, Wen Y, Yang B, Zhang Y, Liu S, Liu Z, et al. Biochemical mechanisms of imidacloprid resistance in Nilaparvata lugens: over-expression of cytochrome P450 CYP6AY1. Insect Biochem Mol Biol. 2013:43(11):1021-7.

65. Joußen N, Agnolet S, Lorenz S, Schöne SE, Ellinger R, Schneider B, et al. Resistance of Australian Helicoverpa armigera to fenvalerate is due to the chimeric P450 enzyme CYP337B3. Proc Natl Acad Sci. 2012;109(38):15206-11.

66. Yang $Y$, Yue $L, C$ Chen $S$, Wu Y. Functional expression of Helicoverpa armigera CYP9A12 and CYP9A14 in Saccharomyces cerevisiae. Pestic Biochem Physiol. 2008:92(2):101-5.

67. Demaeght P, Dermauw W, Tsakireli D, Khajehali J, Nauen R, Tirry L, et al. Molecular analysis of resistance to acaricidal spirocyclic tetronic acids in Tetranychus urticae: CYP392E10 metabolizes spirodiclofen, but not its corresponding enol. Insect Biochem Mol Biol. 2013:43(6):544-54

68. Riga M, Tsakireli D, llias A, Morou E, Myridakis A, Stephanou EG, et al. Abamectin is metabolized by CYP392A16, a cytochrome P450 associated with high levels of acaricide resistance in Tetranychus urticae. Insect Biochem Mol Biol. 2014;46:43-53.

69. Roditakis E, Morou E, Tsagkarakou A, Riga M, Nauen R, Paine M, et al. Assessment of the Bemisia tabaci CYP6CM1VQ transcript and protein levels in laboratory and field-derived imidacloprid-resistant insects and cross-metabolism potential of the recombinant enzyme. Insect Sci. 2011;18(1):23-9.

70. Nauen R, Vontas J, Kaussmann M, Wölfel K. Pymetrozine is hydroxylated by CYP6CM1, a cytochrome P450 conferring neonicotinoid resistance in Bemisia tabaci. Pest Manag Sci. 2013;69(4):457-61.

71. Yang N, Xie W, Yang X, Wang S, Wu Q, Li R, et al. Transcriptomic and proteomic responses of sweetpotato whitefly, Bemisia tabaci, to thiamethoxam. PLoS One. 2013;8(5):e61820.

72. Yang X, Xie W, Wang S-I, Wu Q-j, Pan H-p, Li R-m, et al. Two cytochrome P450 genes are involved in imidacloprid resistance in field populations of the whitefly, Bemisia tabaci, in China. Pestic Biochem Physiol. 2013;107(3):343-50.

73. Markussen MDK, Kristensen M. Cytochrome P450 monooxygenase-mediated neonicotinoid resistance in the house fly Musca domestica L. Pestic Biochem Physiol. 2010;98(1):50-8.
74. Puinean AM, Foster SP, Oliphant L, Denholm I, Field LM, Millar NS, et al. Amplification of a cytochrome P450 gene is associated with resistance to neonicotinoid insecticides in the aphid Myzus persicae. PLoS Genet. 2010;6(6):e1000999.

75. Daborn PJ, Yen JL, Bogwitz MR, Le Goff G, Feil E, Jeffers S, et al. A single P450 allele associated with insecticide resistance in Drosophila. Science. 2002;297(5590):2253-6.

76. Willingham AT, Keil T. A tissue specific cytochrome P450 required for the structure and function of Drosophila sensory organs. Mech Dev. 2004;121(10):1289-97.

77. Karatolos N, Williamson MS, Denholm I, Gorman K, french-Constant RH, Bass C. Over-expression of a cytochrome P450 is associated with resistance to pyriproxyfen in the greenhouse whitefly Trialeurodes vaporariorum. PLoS One. 2012;7(2):e31077.

78. Riaz MA, Poupardin R, Reynaud S, Strode C, Ranson H, David J-P. Impact of glyphosate and benzo[a]pyrene on the tolerance of mosquito larvae to chemical insecticides. Role of detoxification genes in response to xenobiotics. Aquat Toxicol. 2009;93(1):61-9.

79. Tiwari S, Gondhalekar AD, Mann RS, Scharf ME, Stelinski LL. Characterization of five CYP4 genes from Asian citrus psyllid and their expression levels in Candidatus Liberibacter asiaticus-infected and uninfected psyllids. Insect Mol Biol. 2011;20(6):733-44.

80. Nikou D, Ranson H, Hemingway J. An adult-specific CYP6 P450 gene is overexpressed in a pyrethroid-resistant strain of the malaria vector, Anopheles gambiae. Gene. 2003;318:91-102.

81. Bautista MAM, Tanaka T, Miyata T. Identification of permethrin-inducible cytochrome P450s from the diamondback moth, Plutella xylostella (L.) and the possibility of involvement in permethrin resistance. Pestic Biochem Physiol. 2007;87(1):85-93.

82. Amenya DA, Naguran R, Lo TCM, Ranson H, Spillings BL, Wood OR, et al. Over expression of a cytochrome P450 (CYP6P9) in a Major African Malaria Vector, Anopheles Funestus, resistant to pyrethroids. Insect Mol Biol. 2008;17(1):19-25.

83. Zhou X, Sheng C, Li M, Wan H, Liu D, Qiu X. Expression responses of nine cytochrome P450 genes to xenobiotics in the cotton bollworm Helicoverpo armigera. Pestic Biochem Physiol. 2010;97(3):209-13.

84. Le Goff G, Boundy S, Daborn PJ, Yen JL, Sofer L, Lind R, et al. Microarray analysis of cytochrome P450 mediated insecticide resistance in Drosophila. Insect Biochem Mol Biol. 2003;33(7):701-8.

85. Nauen R, Wölfel K, Lueke B, Myridakis A, Tsakireli D, Roditakis E, et al. Development of a lateral flow test to detect metabolic resistance in Bemisia tabaci mediated by CYP6CM1, a cytochrome P450 with broad spectrum catalytic efficiency. Pestic Biochem Physiol. 2015. doi:10.1016/ j.pestbp.2014.1012.1023.

86. Zhuang H-M, Wang K-F, Zheng L, Wu Z-J, Miyata T, Wu G. Identification and characterization of a cytochrome P450 CYP6CX1 putatively associated with insecticide resistance in Bemisia tabaci. Insect Sci. 2011;18(5):484-94.

87. Tsagkarakou A, Tsigenopoulos CS, Gorman K, Lagnel J, Bedford ID. Biotype status and genetic polymorphism of the whitefly Bemisia tabaci (Hemiptera: Aleyrodidae) in Greece: mitochondrial DNA and microsatellites. Bull Entomol Res. 2007;97(01):29-40.

88. Roditakis E, Roditakis NE, Tsagkarakou A. Insecticide resistance in Bemisia tabaci (Homoptera: Aleyrodidae) populations from Crete. Pest Manag Sci. 2005;61(6):577-82.

89. Roditakis E, Tsagkarakou A, Vontas J. Identification of mutations in the para sodium channel of Bemisia tabaci from Crete, associated with resistance to pyrethroids. Pestic Biochem Physiol. 2006;85(3):161-6.

90. Winnebeck EC, Millar CD, Warman GR. Why does insect RNA look degraded? J Insect Sci. 2010;10(159):1-7.

91. FastQC In: http://www.bioinformatics.babraham.ac.uk/index.html.

92. Schmieder R, Edwards R. Quality control and preprocessing of metagenomic datasets. Bioinformatics. 2011;27(6):863-4.

93. scythe In.: https://github.com/vsbuffalo/scythe.

94. SeqPrep. In.: https://github.com/jstjohn/SeqPrep.

95. sickle. In.: https://github.com/najoshi/sickle

96. SILVA rRNA database version 111. In.: http://www.arb-silva.de/

97. fastx_toolkit. In.: http://hannonlab.cshl.edu/fastx_toolkit/index.html

98. Schmieder R, Lim YW, Edwards R. Identification and removal of ribosomal RNA sequences from metatranscriptomes. Bioinformatics. 2011;28(3):433-5.

99. Xie Y, Wu G, Tang J, Luo R, Patterson J, Liu S, et al. SOAPdenovo-Trans: De novo transcriptome assembly with short RNA-Seq reads. Bioinformatics. 2014;30(12):1660-6. 
100. Schulz MH, Zerbino DR, Vingron M, Birney E. Oases: robust de novo RNA-seq assembly across the dynamic range of expression levels. Bioinformatics. 2012;28(8):1086-92.

101. Grabherr MG, Haas BJ, Yassour M, Levin JZ, Thompson DA, Amit I, et al. Full-length transcriptome assembly from RNA-Seq data without a reference genome. Nat Biotech. 2011;29(7):644-52

102. Langmead B, Salzberg SL. Fast gapped-read alignment with Bowtie 2. Nat Methods. 2012;9(4):357-9.

103. Li H, Handsaker B, Wysoker A, Fennell T, Ruan J, Homer N, et al. The sequence alignment/map format and SAMtools. Bioinformatics. 2009;25(16):2078-9.

104. Parra G, Bradnam K, Korf I. CEGMA: a pipeline to accurately annotate core genes in eukaryotic genomes. Bioinformatics. 2007;23(9):1061-7.

105. Lagnel J, Tsigenopoulos CS, lliopoulos I. NOBLAST and JAMBLAST: new options for BLAST and a Java application manager for BLAST results. Bioinformatics. 2009;25(6):824-6.

106. Conesa A, Götz S, García-Gómez JM, Terol J, Talón M, Robles M. Blast2GO: a universal tool for annotation, visualization and analysis in functional genomics research. Bioinformatics. 2005;21(18):3674-6.

107. Hunter S, Apweiler R, Attwood TK, Bairoch A, Bateman A, Binns D, et al. InterPro: the integrative protein signature database. Nucleic Acids Res 2009;37 suppl 1:D211-5.

108. Quevillon E, Silventoinen V, Pillai S, Harte N, Mulder N, Apweiler R, et al. InterProScan: protein domains identifier. Nucleic Acids Res. 2005;33 suppl 2:W116-20

109. Rice P, Longden I, Bleasby A. EMBOSS: the European Molecular Biology Open Software Suite. Trends Genet. 2000;16(6):276-7.

110. Myhre S, Tveit H, Mollestad T, Lægreid A. Additional gene ontology structure for improved biological reasoning. Bioinformatics. 2006;22(16):2020-7.

111. GOSlim. In.: http://geneontology.org/page/go-slim-and-subset-guide

112. Kanehisa M, Goto S. KEGG: Kyoto Encyclopedia of Genes and Genomes. Nucleic Acids Res. 2000;28(1):27-30.

113. Jones A, Brown L, Sattelle D. Insect nicotinic acetylcholine receptor gene families: from genetic model organism to vector, pest and beneficial species. Invert Neurosci. 2007;7(1):67-73.

114. Jones A, Sattelle D. The cys-loop ligand-gated ion channel gene superfamily of the red flour beetle, Tribolium castaneum. BMC Genomics. 2007;8(1):327.

115. Katoh K, Misawa K, Kuma K, Miyata T. MAFFT: a novel method for rapid multiple sequence alignment based on fast Fourier transform. Nucleic Acids Res. 2002;30(14):3059-66.

116. Darriba D, Taboada GL, Doallo R, Posada D. ProtTest 3: fast selection of bestfit models of protein evolution. Bioinformatics. 2011;27(8):1164-5.

117. Stamatakis A. RAxML Version 8: a tool for phylogenetic analysis and postanalysis of large phylogenies. Bioinformatics. 2014;30(9):1312-3.

118. FigTree. In.: http://tree.bio.ed.ac.uk/software/figtree/

119. Koboldt DC, Chen K, Wylie T, Larson DE, McLellan MD, Mardis ER, et al. VarScan: variant detection in massively parallel sequencing of individual and pooled samples. Bioinformatics. 2009;25(17):2283-5.

120. Robinson MD, McCarthy DJ, Smyth GK. edgeR: a bioconductor package for differential expression analysis of digital gene expression data. Bioinformatics. 2010;26(1):139-40.

121. Zhou X, Lindsay H, Robinson MD. Robustly detecting differential expression in RNA sequencing data using observation weights. Nucleic Acids Res. 2014:42(11):e91.

122. Pfaffl MW, Horgan GW, Dempfle L. Relative expression software tool (RESTO) for group-wise comparison and statistical analysis of relative expression results in real-time PCR. Nucleic Acids Res. 2002;30(9):e36.

123. Petersen TN, Brunak S, von Heijne G, Nielsen H. SignalP 4.0: discriminating signal peptides from transmembrane regions. Nat Meth. 2011:8(10):785-6.

124. Krogh A, Larsson B, von Heijne G, Sonnhammer ELL. Predicting transmembrane protein topology with a hidden markov model: application to complete genomes1. J Mol Biol. 2001;305(3):567-80.

doi:10.1186/s12864-015-2161-5

Cite this article as: llias et al:: Transcription analysis of neonicotinoid resistance in Mediterranean (MED) populations of $B$. tabaci reveal novel cytochrome P450s, but no nAChR mutations associated with the phenotype. BMC Genomics 2015 16:

\section{Submit your next manuscript to BioMed Central and take full advantage of:}

- Convenient online submission

- Thorough peer review

- No space constraints or color figure charges

- Immediate publication on acceptance

- Inclusion in PubMed, CAS, Scopus and Google Scholar

- Research which is freely available for redistribution 